September 20, 1998

\title{
Computer Simulations of Supercooled Liquids and Glasses*
}

\author{
Walter Kob \\ Institut für Physik, Johannes Gutenberg-Universität, Staudinger Weg 7, D-55099 Mainz, \\ Germany
}

\begin{abstract}
After a brief introduction to the dynamics of supercooled liquids, we discuss some of the advantages and drawbacks of computer simulations of such systems. Subsequently we present the results of computer simulations in which the dynamics of a fragile glass former, a binary Lennard-Jones system, is compared to the one of a strong glass former, $\mathrm{SiO}_{2}$. This comparison gives evidence that the reason for the different temperature dependence of these two types of glass formers lies in the transport mechanism for the particles in the vicinity of $T_{c}$, the critical temperature of mode-coupling theory. Whereas the one of the fragile glass former is described very well by the ideal version of mode-coupling theory, the one for the strong glass former is dominated by activated processes. In the last part of the article we review some simulations of glass formers in which the dynamics below the glass transition temperature was investigated. We show that such simulations might help to establish a connection between systems with self generated disorder (e.g. structural glasses) and quenched disorder (e.g. spin glasses).
\end{abstract}

PACS numbers: 61.20.Lc, 61.20.Ja, 02.70.Ns, 64.70.Pf

Typeset using REVTEX

*To appear as a Topical Review Article in J. Phys.: Condens. Matter 


\section{INTRODUCTION}

The history of man-made glasses is several thousand years old [1] and thus it might seem that we had enough time to gain an excellent understanding of this type of material. A brief glance at the recent literature and the topic of many specialized conferences shows, however, that this is not the case at all. Although a vast amount of detailed knowledge on various properties of all sorts of glasses has been accumulated, and our understanding of these materials has certainly increased tremendously since ancient times, the answer to some of the most basic issues are still a matter of debate. Apart from some questions that have been posed already many years ago, such as regarding the mechanism that gives rise to the glass transition, new questions have emerged very recently, such as, e.g., the nature of the glass transition in small pores, whether or not glasses are dynamically homogeneous or heterogeneous, or how systems with frozen in disorder (e.g. spin glasses) are related to the ones with self generated disorder (e.g. structural glasses). Because of the vastness of the field the present article does of course not even attempt to give an exhaustive review on all these different topics and developments and we refer the interested reader to the various textbooks and review articles on that subject [2 - 1 . In the following we will therefore focus on only certain topics of supercooled liquids and glasses. Hence the fact that many other issues will be treated only briefly or not at all should not be viewed as a statement of their irrelevance but rather as a (somewhat arbitrary) choice of the author.

Computer simulations have of course a much shorter history than glasses since the first work dates back only to the 1950's [5]. However, by now it has been shown that such simulations can be an excellent tool to investigate the properties of complex systems [6] and it can be expected that with the availability of faster and cheaper computers, as well as improved algorithms, such simulations will play an even more important role in the future than they do now. In this article we will discuss how such simulations can be used to gain insight into the structure and the dynamics of supercooled liquids and glasses. Again, the work on this subject is by now far too extensive to be completely covered in this article and therefore we present only a small, but hopefully relevant, subset of it and refer the interested reader to the mentioned textbooks [2, 3] and other review articles on this subject [4, [7,8].

In order to facilitate the reading of the articles for those not familiar with the subject we will give in Sec. II a brief introduction to the field of supercooled liquids and glasses and discuss some of the questions that are currently debated. The following section is then devoted to issues related to computer simulations. In Sec. IV we will discuss results regarding the equilibrium dynamics of fragile and strong glass formers and at the end show that also in the non-equilibrium dynamics of glass forming liquids many very interesting phenomena occur, which so far are not understood at all, and which can be studied very well by means of computer simulations.

\section{SUPERCOOLED LIQUIDS AND GLASSES}

In this section we will briefly review some facts regarding the structure and dynamics of supercooled liquids and glasses. Despite its briefness it should allow the reader to get familiar with some of the issues concerning supercooled liquids and glasses so that the 
following sections become more comprehensible. More detailed discussions to these topics can be found in Refs. 2 -4.

First of all it is appropriate to specify what we mean in the following by the terms "supercooled liquids" and "glass". The standard point of view is the following: If a liquid can be cooled below its melting temperature $T_{m}$ without the occurrence of crystallization, it is called a good glass former, and when the temperature is less than $T_{m}$ the system is called supercooled. The static and dynamical properties of such systems can be studied in a large temperature range below $T_{m}$ and it is found that their relaxation times increase very fast by many (12-14) decades if the temperature is lowered. At a certain temperature the relaxation time exceeds the time scale of the experiment and therefore the system will fall out of equilibrium. It is this falling out of equilibrium what is called the glass transition. At temperatures well below this glass transition temperature no relaxation seems to take place anymore (on any reasonable time scale) and it is customary to call this material a glass. (Note that this transition temperature will in general depend on the type of experiment since its definition involves the time scale of the experiment. This definition should also not be confused with the one often used by experimentalists in which a glass transition temperature is defined as the temperature at which the viscosity of the system has the (somewhat arbitrary) value $10^{13}$ Poise.)

In the following we will adapt a point of view on "supercooled" which is slightly different and is motivated by the experimental observation that if a system approaches its glass transition temperature its relaxation dynamics becomes non-Debye, i.e. that the time correlation functions decay in a non-exponential way (e.g. they show a two-step relaxation), whereas it shows a Debye-behavior at high temperatures. The reason for this phenomenon will be discussed below. It has been found, however, that, e.g., Glycerol and $\mathrm{B}_{2} \mathrm{O}_{3}$ show this sort of non-Debye behavior also at temperatures significantly above the melting temperature [9, 10]. Therefore one has to conclude that the non-Debye relaxation has nothing to do with the system being supercooled, a point of view which is supported from the theory of the dynamics of dense liquids [11]. In addition it is not hard to imagine a system that does not crystallize at all, i.e. one for which the concept of a melting temperature, and hence the term "supercooled", does not even exist (e.g. in atactic polymers). Nevertheless it can be expected that the dynamics of such a system will become very slow when the temperature is decreased and that thus the system will undergo a glass transition. For these reasons we will mean in the following by "supercooled" that the relaxation dynamics of the system is non-exponential and not that its temperature is below $T_{m}$. Furthermore, in order to simplify the language, we will use in the following always temperature as the variable that drives the slowing down of the dynamics. The reader should, however, bear in mind that there are systems in which the glass transition is driven by a change of particle concentration, such as colloids or kinetic lattice gases 12,13.

If the structural and dynamical properties of a good glass former are measured in the temperature range between the high temperature regime and the glass transition temperature, one finds that all structural quantities (density, structure factor, etc.) and thermodynamic quantities (specific heat, etc.) show a very gentle temperature dependence, in that they change between a few percent or a factor of 2-3 [2, 3]. (Note that the experimental observation that the specific heat $C_{p}$ shows a pronounced drop at the glass transition temperature, see, e.g., Ref. [14] does not contradict this statement, since this effect is due to fact that 
the system falls out of equilibrium, i.e., that certain translational degrees of freedom do not contribute anymore to the specific heat. Thus the drop in $C_{p}$ is a purely kinetic effect.) As already mentioned above, dynamic quantities, such as the diffusion coefficient $D$ or the viscosity $\eta$, will in general show a much more pronounced temperature dependence than thermodynamic quantities. It is this huge variation of the transport coefficients that makes the experimental investigation of the dynamics, and its theoretical description, such an interesting challenge. Phenomenologically the temperature dependence of the dynamics can be described quite well by the so-called Vogel-Fulcher law $\eta \propto \exp \left(E T_{0} /\left(T-T_{0}\right)\right)$, where $T_{0}<T_{g}$ is the so-called Vogel temperature and $E$ is a parameter that determines the shape of the curve. If $E$ is large the temperature dependence is Arrhenius like and if it is small, $\eta$ shows a pronounced upward bend at a temperature a bit above $T_{0}$. Angell coined the terms "strong" and "fragile" glass formers for the former and latter case, respectively [15]. Below we will discuss how the dynamics of strong and fragile glass formers differ on the microscopic level.

Having discussed some of the phenomena observed in the temperature dependence of glass forming liquids it might be useful at this point to make some comments on the various theoretical approaches that have been used to rationalize the dramatic slowing down of the dynamics when the temperature is lowered. One of the most simple potential mechanisms is, that upon cooling the system approaches the critical point of a second order phase transition and hence the increase of the relaxation times is just the critical slowing down of the system when the temperature approaches this point. It is generally believed that it is this mechanism that is responsible for the glass transition in spin glasses [ [4, 16 [18]. For structural glasses the situation is much less clear since the presence of a second order thermodynamic phase transition implies that one should be able to identify an order parameter or a growing length scale. Since in computer simulations it is relatively easy to measure such an order parameter or length scale, if one knows what to look for, they have been used quite extensively to find evidence for the existence of a thermodynamic transition in structural glasses. However, as discussed in detail in Ref. [19], these efforts have led to no positive results so far.

One of the first successful theories for the glass transition is the one proposed by Gibbs and DiMarzio [20 for dense polymer melts. The basic idea of this theory is that with decreasing temperature the configurational entropy to the polymers decreases and vanishes at a finite temperature, thus leading to the glass transition. Below we will come back to this theory and discuss it more closely in the context of some computer simulations that have been performed to check its validity.

Another very successful theory is the so-called mode-coupling theory (MCT) which has been worked out by Götze, Sjögren and coworkers [11]. Starting from the theory of dense simple liquids [21] MCT derives equations of motion for time and wave-vector dependent correlation functions and makes very detailed predictions on the time and temperature dependence of these quantities when the system is in the supercooled state. As will be discussed below, many of the predictions of this theory have been confirmed in experiments and computer simulations and thus MCT can presently be regarded as the best available theory of the dynamics of supercooled liquids.

In the following sections we will extensively discuss computer simulations that have been done in order to test the validity of the two theories just mentioned. The goal of this discussion is not to give an exhaustive review of all the possible tests that have been done 
so far, but rather to present some exemplary results of simulations in order to convince the reader that such simulations can be a very powerful tool to check to what extend a theory is valid or not.

\section{COMPUTER SIMULATIONS OF GLASS FORMERS: ADVANTAGES AND DRAWBACKS}

The goal of this section is to discuss certain aspects of computer simulations that are particular to simulations of supercooled liquids and glasses. More general introductions to simulations can be found in various textbooks, such as Refs. [6].

The most outstanding advantage of computer simulations is that they provide an extremely large freedom regarding the systems that can be studied. In principle, it is no problem to investigate any Hamiltonian that one is interested in, be it on a classical or quantum mechanical level. By a judicious choice of the Hamiltonian considered, it is therefore possible to investigate, e.g., systems in which the dynamics is determined by purely kinetic constraints instead by energetic ones [13,22,23, , to study molecules or polymers with an exactly specified shape and size [24 27], to investigate the system in thermodynamics states which are difficult to realize experimentally, such as negative pressures and high temperatures 28 32], or the dependence of the structure and the dynamics of a system in a small pore of a well-defined size and shape [33,34]. For example simulations of supercooled water have given evidence that this system has a liquid to liquid transition [35] and from simulations of $\mathrm{SiO}_{2}$ novel (crystalline) phases have been predicted [36].

In addition to this, simulations offer the unique possibility to access any observable of interest, since the complete information on the positions and velocities of all the particles is available at any given time. This property of simulations allows to determine quantities which are very difficult to access in real experiments or very hard to obtain with reasonable precision in an analytical calculation. Hence it is possible to use such simulations to test theoretical concepts and theories in a more stringent way than it is feasible with real experiments. Examples for such type of simulations will be discussed below.

Despite all these advantages reality is not quite as rosy as it might seem since one is faced with the sad fact that computer resources are finite. Therefore it is, e.g., presently out of question to do a full quantum mechanical simulation for several thousand particles over a time scale of, e.g., $1 \mathrm{~ns}$, and even with classical force fields and $O\left(10^{3}\right)$ particles it is hard to simulate times significantly longer than $100 \mathrm{~ns}$, since each time step is usually only $10^{-15} \mathrm{sec}$ long (for atomic systems). The present state of the art is to make an ab initio calculation of about 100 particles for a time span of 10 ps [37,38] whereas for classical simulations one can deal with box sizes of 50-100Åand simulate such a system for 10-100 ns [39,40]. (Such a simulation will then usually take the equivalent of several CPU years on a very good, dedicated workstation.) Of course one might wonder whether it really is necessary to study systems that are larger than a few hundred particles, since it is possible to study many aspects of the glass transition even with such small systems. However, there exist situations in which large systems are necessary. E.g. the dynamics of strong glass formers shows quite pronounced finite size effects [40], and the same is true also for the density of states in the frequency range which is important for the so-called boson-peak, a dynamic feature at low energies whose nature is currently a matter of intense debate [41,42]. 
Note that, despite the mentioned limitations in time and system size, large scale computer simulations cover a wave-vector and frequency range which is comparable or even larger than, e.g., the one accessible in neutron-scattering experiments and hence they can be a valuable addition to investigate the structure and dynamics of systems on this length and time scale. However, it has to be emphasized that real experiments still have a crucial advantage over simulations, namely in the way the sample is prepared. What experimentalist do is to adjust the temperature of the sample to the temperature of interest and wait on the order of minutes or even weeks before they start to do the measurement. Therefore it becomes possible to probe the equilibrium dynamics of the system on the ns scale (e.g. in the case of neutron scattering) even if the relaxation time of the system is on the order of days. In computer simulations such a procedure is (not yet) possible. The only way to equilibrate the system is to do a simulation at the temperature of interest for a time span that is on the order of the longest relevant relaxation time of the system and to subsequently start the run for the production. Since typical time spans of the simulation do not exceed $100 \mathrm{~ns}$, it is therefore only possible to equilibrate the system at a relatively high temperature. Thus all equilibrium measurements are restricted to these temperatures as well.

In order to circumvent this problem one might be tempted to quench the system relative rapidly to a temperature at which its relaxation time is larger than the one accessible to computer simulations, let the system relax for some time, and subsequently start to measure its properties. Such an approach is, however, quite dangerous in that the results from such a simulations will in general show aging effects. This means that quantities that should be constant (such as the average potential energy) show a small drift, and that time correlation functions will no longer be time-translation invariant. Some of these effects will be discussed in more detail in Sec. IVB.

We stress, however, that the above mentioned problem with the equilibration of the sample is not a principle one. There is no reason why a cleverly designed Monte Carlo algorithm should not be able to equilibrate the sample also at a temperature at which, e.g., the relaxation time for the usual Newtonian dynamics is macroscopically large. Examples for such algorithms have already been successfully implemented for polymers and it was found that they allow to equilibrate the sample about 100 times faster than with the normal dynamics 43. In recent years other methods have been proposed and tested and, although presently there still is no optimal method in sight, the progress is quite encouraging [44] and therefore it can be hoped that sooner or later this bottleneck will be removed.

Before we end this section it is appropriate to make some comments on the Hamiltonians used in simulations of supercooled liquids and glasses. Roughly speaking simulations in this field can be divided into two types:

i) In the first one the main goal is to use the simulation to gain an understanding on some very general question, such as to what extend $\mathrm{MCT}$ gives a correct description of the dynamics, the nature of the dynamical heterogeneities observed in experiments [45.46], or the search for a diverging length scale when the temperature approaches $T_{g}$ [33, 47]. Since one is interested in some universal properties of glassy systems, such simulations are usually done with very simple systems, such as Lennard-Jones particles, kinetic Ising models [13] or even simpler ones (see, e.g., the backgammon model of Ritort [22]). Because of their simplicity such models allow to obtain results of a much higher accuracy than it would be possible with more realistic and thus more complicated systems and thus more definite 
answers can be given to the question of interest.

ii) In the second type of simulation one attempts to answer some quite specific questions for a given material (or class of materials), such as, e.g., the nature of the so-called bosonpeak in strong glass formers [41,42,48, 50, to identify the mechanism leading to the so-called "mixed alkali effect" [51], or to determine the distribution function of the rings in network forming systems such as $\mathrm{SiO}_{2}[38,52$ 54]. For such simulations is is important to have a reliable potential at hand which is able to give a sufficiently realistic description of the quantity that one is interested in. Unfortunately it is still the exception rather than the rule that for the material of interest a good potential is available. The reason for this is that the development of a reliable force field often involves a substantial amount of $a b$ initio calculations, in order to determine the potential energy of some typical configurations, and also the subsequent fit of these data points to a classical potential energy function is often rather difficult (see, e.g., the papers of Takada et al. [55]). Both of these steps require a substantial amount of expertise and work and thus have been done only for a few selected substances, such as, e.g., silicates [56-59] or $\mathrm{ZnCl}_{2}$ 60.

It should also be noted that whether or not a potential is realistic depends on the question one is interested in. For the case of silica, e.g., there exist many different potentials, many of which give a quite realistic representation of the structural properties of amorphous $\mathrm{SiO}_{2}$, such as the structure factor 8, 28, 36, 42,52 54,61, 64]. Thus from this point of view the various potentials can be considered as essentially equivalent. If, however, dynamical quantities are considered, such as the diffusion constant, one finds that the various potentials make very different predictions. In Fig. 10 we reproduce data by Hemmati and Angell in which the temperature dependence of the oxygen-diffusion constant is shown for different potentials [61]. One sees that at high temperatures the values of the diffusion constants predicted by the different potentials differ only by about a factor of three. This agreement changes dramatically when the temperature is lowered in that the different models predict diffusivities that vary over several orders of magnitude. Hence we conclude that a potential that might be appropriate to describe the structure of $\mathrm{SiO}_{2}$ might be unsuitable for describing the dynamics of the system.

\section{SOME EXAMPLES OF COMPUTER SIMULATIONS OF SUPERCOOLED LIQUIDS AND GLASSES}

In this section we will discuss some computer simulations of supercooled liquids and

glasses. In the first part we will make a comparison between the equilibrium dynamics of a fragile glass former and a strong glass former in order to see what the similarities and differences in these two types of systems are. In the second part we will discuss the nonequilibrium (aging) dynamics of a simple glass former, i.e. the decay of the time correlation functions of the system after it has been subjected to a quench to a low temperature.

\section{A. The relaxation dynamics of strong and fragile glass formers}

We already mentioned at the beginning of this article that the temperature dependence of transport quantities of glass forming liquids is not universal in that certain glass formers 
(called "strong") show an Arrhenius dependence and others (called "fragile") show strong deviations from it [15]. In this subsection we will present the results of computer simulations in which the dynamics of a simple fragile glass former and $\mathrm{SiO}_{2}$, a prototype of a strong glass former was investigated. By a comparison between the two dynamics we will attempt to understand what the difference between the two types of glass formers are on the microscopic level.

Very often fragile glass formers are van der Waals liquids in which the interactions between the (small) molecules is relatively weak. Thus such systems can be modelled by particles with isotropic short range interactions such as soft spheres, $V(r) \propto r^{-12}$, or the Lennard-Jones potential. Because of the simplicity of these potentials the relaxation dynamics of such models has been studied extensively by means of computer simulations [19, 65 ]73]. Early simulations often focussed on one-component systems [74] but with the increased speed of the computers it was found that such systems started to crystallize within the time span of the simulation. A simple way to avoid this problem is to use a binary mixture of particles and if the interaction parameters and the concentration of the species is chosen well, such systems stay in the (meta)stable liquid-like state for all time spans which a present state of the art simulation can cover, i.e. over 100 million time steps, which corresponds to about $100 \mathrm{~ns}$. A binary mixture of soft spheres has, e.g., been investigated in great detail by the group of Hansen [66], work that has been continued by Hiwatari, Odagaki and coworkers [67, and very recently this system has been used by Parisi and coworkers to investigate aging phenomena 69, 70.

The binary system we will discuss here consists of an 80:20 mixture of Lennard-Jones particles, $V_{\alpha \beta}(r)=4 \epsilon_{\alpha \beta}\left[\left(\sigma_{\alpha \beta} / r\right)^{12}-\left(\sigma_{\alpha \beta} / r\right)^{6}\right]$, where $\alpha, \beta \in\{A, B\}$ denotes the type of the particle ( $A$ being the majority species). The parameters of the potential are given by $\sigma_{A A}=1.0, \epsilon_{A B}=1.5, \sigma_{A B}=0.8, \epsilon_{B B}=0.5$, and $\sigma_{B B}=0.88$. For this system we will report length in units of $\sigma_{A A}$, and energy and time in units of $\epsilon_{A A}$ (setting $k_{B}=1$ ) and $\left(m \sigma_{A A}^{2} / 48 \epsilon_{A A}\right)^{1 / 2}$, respectively, where $m$ is the mass of the particles. In the simulation we used a cubic box, of length 9.4, with periodic boundary conditions and the total number of particles was 1000, which is large enough to avoid finite size effects almost completely. More details on these simulations can be found in Refs. 772,73.

As already mentioned above, there exist quite a few different potentials for $\mathrm{SiO}_{2}$ (see, e.g., the references in [54]). The one proposed by van Beest, Kramer and van Santen (BKS) [57] seems to be one of the best, in that it is able to reproduce well many of the structural and dynamical features of real silica. In this potential the interactions $\phi_{i j}$ between ions $i$ and $j$ at a distance $r$ apart is given by:

$$
\phi_{i j}(r)=\frac{q_{i} q_{j} e^{2}}{r}+A_{i j} \exp \left(-B_{i j} r\right)-\frac{C_{i j}}{r^{6}} .
$$

Here $e$ is the charge of an electron and the constants $A_{i j}, B_{i j}$ and $C_{i j}$ can be found in Refs. [54,57. One interesting aspect of this potential is that it contains only two-body terms. This is somewhat surprising since $\mathrm{SiO}_{2}$ forms an open tetrahedral network and thus it might be expected that three-body terms are needed as well. However, it has been shown that the BKS potential is indeed able to generate such a (disordered) tetrahedral network [54], since the competition between the different two-body forces mimic the threebody forces. As a side remark we mention that the absence of the three-body terms is of 
course advantageous for computer simulations, since their evaluation is usually demanding from a computational point of view. Thus the only part of the potential whose calculation is computationally intensive is the long-range Coulombic part, which is usually calculated by means of the Ewald summation [6,75]. Since the computational effort of this method scales with $N^{3 / 2}$, where $N$ is the number of particles, it is clear that doing simulations of large systems with long range potentials is computationally much more demanding than if the potential is short ranged (and whose computational effort thus scales like $N$ ). However, since a few years new methods have become available in which the calculation of the long ranged forces also (essentially) scales like $N$ [76]. The disadvantage of these methods is that they become efficient only at relatively large $N$, and thus for small systems (a few hundred particles) the Ewald summation is presently the only real possibility to handle such forces. In practice this means that for a system of the order of $10^{3}$ particles the calculation of the forces on all the particles in a system with Coulombic interactions is about one order of magnitude more time consuming than in the case of short range interactions (e.g. Lennard-Jones).

Since it has been found that the dynamics of strong glass formers shows quite large finite size effects [40] it is necessary to use for such simulations relatively large systems sizes. In the following we will discuss results of simulations in which 8016 ions have been used, which corresponds to a box size of about $48 \AA$. More details on the simulation can be found in Refs. 40,64,77.

Before we start the discussion of the dynamical behavior of the two glass formers, we will have a brief look at the temperature dependence of the static structure of these systems. One possibility to do this is to investigate the partial structure factors $S_{\alpha \beta}(q)$ which are given by $\left\langle\rho_{\alpha}(q) \rho_{\beta}^{*}(q)\right\rangle$, where $\rho_{\alpha}(q)$ is the fluctuation of the density of particles of type $\alpha$ for wavevector $q$, i.e. $\rho_{\alpha}(q)=\sum_{j=1}^{N_{\alpha}} \exp \left(i \mathbf{q} \cdot \mathbf{r}_{j}^{\alpha}\right)$, and $\mathbf{r}_{j}^{\alpha}$ is the position of the $j$ th particle of type $\alpha$. In Fig. 2 we show the partial structure factors for the $A-A$ and oxygen-oxygen correlations, for the Lennard-Jones and silica system, respectively. The temperatures for the different curves range from temperatures at which the system is in its normal liquid state to temperatures at which the system is in a deeply supercooled state. From the figure it can be seen that the temperature dependence of this structural quantity is rather weak and that the main effect is that the various peaks and minima become more pronounced when the temperature is lowered. Although the static structure factors do not depend strongly on temperature, time dependent quantities do show a strong dependence. In order to demonstrate this we have included in the figure also the diffusion constant at the different temperatures. From these figures one recognizes that in the temperature range considered, the dynamics of the system does indeed slow down quite dramatically. We will discuss this point in more detail below.

One comment on the meaning of the various peaks might be useful. In the case of a simple liquid, Fig. 2a, the first peak in the structure factor corresponds to the typical interparticle distance. This is in contrast to the case of network-forming systems, Fig. 2 $\mathrm{b}$, where this distance corresponds to the second peak in $S(q)$. The first peak in the structure factor, often called first sharp diffraction peak [2], is related to the size of the structural units making up the network (i.e. tetrahedra in the case of $\mathrm{SiO}_{2}$ ). It is interesting to see that this peak starts to be visible already at the highest temperature investigated. This means that the network is forming already at temperatures much higher than the melting temperature, which is around $2000 \mathrm{~K}$. This is probably the reason why network-forming systems are often very viscous even at very high temperatures. 
It has to be emphasized that the observed (unspectacular) temperature dependence of the structure factor is not exceptional for structural quantities. Although several efforts have been made to find a structural quantity that shows a more pronounced temperature dependence, no clear evidence has been found so far 33,47. Thus the point of view that the slowing down of the dynamics of structural glasses is related to a second order phase transition, and hence to the existence of a divergent length scale, is so far not supported by good evidence. (See also Ref. [79] on this point.)

A much more interesting dependence on temperature than the one for structural quantities is found in time dependent correlation functions, or transport coefficients. The simplest example is $\left\langle r_{\alpha}^{2}(t)\right\rangle$, the mean squared displacement (MSD) of a tagged particle of type $\alpha$ :

$$
\left\langle r_{\alpha}^{2}(t)\right\rangle=N_{\alpha}^{-1} \sum_{i=1}^{N_{\alpha}}\left\langle\delta\left(r^{2}-\left|\mathbf{r}_{i}^{\alpha}(t)-\mathbf{r}_{i}^{\alpha}(0)\right|^{2}\right)\right\rangle
$$

where $\langle$.$\rangle is the thermal average. The time dependence of the MSD for different temperatures$ is shown in Fig. 3. Let us first consider the MSD for the Lennard-Jones system. At high temperatures, top curves, the MSD shows at short times a quadratic dependence on time, $\left\langle r_{\alpha}^{2}(t)\right\rangle \propto t^{2}$. This behavior can be understood immediately by realizing that for short times the particles will move ballistically, i.e. $\mathbf{r}_{i}^{\alpha}(t) \approx \mathbf{r}_{i}^{\alpha}(0)+\dot{\mathbf{r}}_{i}^{\alpha} t$, and thus give rise to the observed time dependence for $\left\langle r_{\alpha}^{2}(t)\right\rangle$. For longer times the particles start to collide with their neighbors and their motion becomes diffusive. Therefore the MSD shows a linear dependence on time, as can be seen in Fig. 3. For low temperatures (bottom curves) the situation at short and very long times is similar to the one at high temperatures in that the ballistic and the diffusive behavior are observed. For intermediate times, however, the MSD shows a feature not present at high temperatures, namely a plateau. This means that there exists a time range, which at the lowest temperatures extends over several decades, in which the MSD does not increase substantially. The microscopic reason for this plateau is that the tagged particle is trapped in the cage formed by the neighboring particles that surround it, and it takes the particle a long time to escape this cage. Note that the particles forming this cage are of course sitting in cages as well and thus the motion of all particles is slowed down. With decreasing temperature the cages become more and more rigid and thus the time needed to break them up increases. The earlier mentioned MCT [11] is an attempt to describe this breaking up in a self consistent way and hence to rationalize the dramatic increase of the relaxation time on a microscopic level.

In Fig. $3 \mathrm{~b}$ we show the MSD for the oxygen atoms in the silica melt. We recognize that for this strong glass former the curves look qualitatively similar to the ones of the fragile glass former. The main difference is that at low temperatures the MSD for silica shows a little bump at around $0.2 \mathrm{ps}$ 64, 80]. The reason for this feature lies in the so-called boson-peak, a dynamical feature whose intensity seems to be related to the fragility of the glass [10], and which will be discussed in more detail below.

Using the Einstein relation $D=\lim _{t \rightarrow \infty}\left\langle r^{2}(t)\right\rangle / 6 t$, the diffusion constant $D$ can be calculated from the MSD. Before we discuss the temperature dependence of $D$ it is useful to review a few of the predictions of MCT [11] since they will be helpful to understand the following results. As already mentioned before, this theory attempts to describe the dynamics of supercooled liquids in a self-consistent way. Most of the predictions of the theory have been worked out for that version of MCT in which certain terms in the equations of the 
theory, the so-called hopping terms, are neglected. This special case is called "ideal MCT" and it is predicted that there exist a special "critical" temperature $T_{c}>0$ in the vicinity of which the dynamics shows an anomalous temperature dependence, in that the relaxation times $\tau$, or the inverse of the diffusion constants, show a power-law divergence, $\tau \propto D^{-1} \propto$ $\left(T-T_{c}\right)^{-\gamma}$. In this ideal case the system does not relax anymore if the temperature is below $T_{c}$. If the mentioned hopping terms are taken into account the divergence does not really take place. If these terms are weak the relaxation times will show the mentioned powerlaw, but slightly above $T_{c}$ they will change over to an Arrhenius law 81]. Empirically it is found that fragile (and not so fragile) glass formers can be described well by the idealized MCT [11, whereas not too much is known about strong glass formers.

We now discuss the temperature dependence of the diffusion constants, which are shown in Fig. 1. Note that we use two different types of plots to present the data for the LennardJones and the silica system. For the former system, a fragile glass former, we have fitted the low-temperature data with a power-law in order to check whether the mentioned temperature dependence predicted by MCT gives a good fit to the diffusion constant. Using $T_{c}$ as a fit parameter we find that this is indeed the case (see Fig. 国a). The critical temperature $T_{c}$ is the same for the $A$ and $B$ particles, in agreement with the prediction of MCT. According to the theory, also the value of the exponent $\gamma$ should be independent of the particle species, and we find that this is reasonably well fulfilled in that for our system the two value agree to within $15 \%$ (see figure).

We also mention that such power-laws have also been found in other simulations of simple liquids [25,66. 21], polymeric systems [26], a simple molecular liquid [27], and water 83. The result on water is of particular interest since $\mathrm{H}_{2} \mathrm{O}$ is a network forming liquid, thus structurally very similar to silica, the system we will discuss next.

For silica the temperature dependence of $D$ is more complicated than the one of fragile glass formers. Since this is a strong glass former, we expect that at low temperatures an Arrhenius behavior is found and thus it is reasonable to plot the data versus $1 / T$, which is done in Fig. 四 b. From the figure we see that at low temperatures the expected Arrhenius law is indeed observed and that the activation energies are close to the ones found in experiments [82 (see figure). Thus we have evidence that our model for silica is quite realistic not only regarding static quantities [54,77] but also dynamical ones, at least at low temperatures.

At higher temperatures strong deviations from the Arrhenius law are observed in that the temperature dependence is weaker than the one expected from the activated dynamics at low $T$. In this temperature range the curves can be fitted well with a power-law, as predicted by $\mathrm{MCT}$, with a critical temperature which is independent of the species and has a value of about $3330 \mathrm{~K}$ (see figure). (We also mention that the given values of $\gamma$ are compatible with the one from the MCT analysis of the $\beta$-relaxation regime.) Thus from the temperature dependence of the diffusion constants we have evidence that also the strong glass former $\mathrm{SiO}_{2}$ shows at high enough temperatures the non-Arrhenius dependence observed for fragile glass formers. This result is in agreement with findings of Rössler and Sokolov [84. By analyzing experimental viscosity data, these authors arrived to the conclusion that for all glass formers there exist a temperature range in which the temperature dependence of the transport coefficients is non-Arrhenius. Hence we conclude that the main difference between strong and fragile glass formers is that in the former the hopping processes that destroy the 
dynamical singularity predicted by the ideal version of MCT are so strong, that the signature of this singularity, namely the power-laws, accounts only for a relatively small range in the diffusion constant (or other transport quantities) before the hopping processes take over and lead to an activated dynamics. In contrast to this are the fragile systems in which the mentioned power-law can be observed over several decades in the diffusion constant.

This point of view is also corroborated by the analysis of the self part of the van Hove correlation function $G_{s}(r, t)$ [21]. This function, or rather $4 \pi r^{2} G_{s}(r, t)$, gives the probability that a particle has moved a distance $r$ in time $t$. It is found that for fragile glass formers, such as the present Lennard-Jones system, this function decreases monotonically as a function of $r$ [73] whereas for silica at intermediate and low temperatures the distribution function for the oxygen atoms show a secondary peak at a value of $r$ which corresponds to the typical oxygen-oxygen distance (no secondary peak is found for the silicon atoms) [77]. From the existence of such a secondary peak it has been concluded [66,71] that the transport mechanism is not the flow-like motion described by MCT but more a jump-like motion that is activated. This point of view is thus in agreement with the one put forward above for the motion of the atoms in silica.

Experimentally it is not possible to measure, for atomic systems, time and space correlation functions like $G_{s}(r, t)$. However, in neutron time of flight measurements it is possible to study its space Fourier transform, the incoherent intermediate scattering function $F_{s}(q, t)$ [21],

$$
F_{s}(q, t)=\frac{1}{N_{\alpha}} \sum_{j=1}^{N_{\alpha}}\left\langle\exp \left(i \mathbf{q} \cdot\left[\mathbf{r}_{j}^{\alpha}(t)-\mathbf{r}_{j}^{\alpha}(0)\right]\right\rangle,\right.
$$

and in neutron and light scattering experiments one has access to its space and time Fourier transform, the dynamical structure factor $S(q, \omega)$. Therefore it is interesting to calculate these quantities from the simulations as well. (We note, however, that an accurate calculation of $S(q, \omega)$ from a simulation is rather difficult, since calculating the time Fourier transform of a correlation function that extends over 6-8 decades in time is not a simple task. Therefore results are usually presented in the time domain.)

In Fig. [5 we show the time dependence of the intermediate scattering function for different temperatures. Let us first discuss the relaxation dynamics for the fragile glass former, Fig. Fa. The wave-vector $q$ corresponds to the location of the first maximum in the structure factor (see Fig. 2a a) but we have found that for the other values of $q$ a qualitatively similar time and temperature dependence is found [72]. After the ballistic motion of the particles at short times, giving rise to a quadratic dependence of $F_{s}(q, t)$ on time, the correlator shows at high temperatures a crossover to an exponential decay. For low temperatures we find a different relaxation behavior in that, after the microscopic regime, the correlation functions show a plateau, the length of which increases rapidly with decreasing temperatures. The reason for the existence of this plateau is the same one we gave in the discussion of the MSD in Fig. 3, namely the cage effect, i.e. the temporary trapping of the particles by their neighbors. It is customary to call the time window in which the correlator is close to the plateau the " $\beta$-relaxation regime" and the window in which the correlator falls below the plateau the " $\alpha$-relaxation regime".

From the figure we also recognize that at low temperatures the shape of the curves does, in the $\alpha$-relaxation regime, not depend on temperature, an observation that we will discuss 
in more detail below. Thus the whole increase of the relaxation times is due to the dynamics in the $\beta$-relaxation regime and hence it is this regime which has to be understood from a theoretical point of view in order to give a correct description of the relaxation dynamics, and hence the glass transition.

For the strong glass former the time dependence of the correlation functions is qualitatively similar to the one of the fragile glass former. In Fig. [5 b we show $F_{s}(q, t)$ for the oxygen atoms for a wave-vector at the location of the first sharp diffraction peak. The main, readily observable, difference between the relaxation behavior of the fragile and the strong glass former is that at low temperatures the latter shows a dip at around $0.2 \mathrm{ps}$, i.e. shortly after the microscopic regime and before the correlator shows the plateau. The time at which this dip occurs corresponds to about $1.5 \mathrm{THz}$, a frequency at which silica shows a pronounced enhancement of the density of states over the value expected from a Debye law [41]. Therefore this feature is called the "boson-peak" ("boson" because its temperature dependence is given by the Bose-factor). Note that this feature is observable already at $T=3580 \mathrm{~K}$, thus at temperatures far above the (experimental) glass transition temperature of silica, which is 1450K. (We also mention that in Ref. [77] evidence is given that the glass transition temperature of the BKS model is very close to this experimental value, thus giving further support for the reliability of this potential.) The nature of the excitations leading to the boson-peak is still a matter of debate [41,42,49] and we do not enter that discussion here. (The issue is on whether the peak is due to localized modes or due to a strong scattering of sound waves). We mention, however, that it was found from computer simulations of systems with different sizes, that the depth of the dip in $F_{s}(q, t)$ depends strongly on the size of the system and that these finite size effects become more severe with decreasing temperature [40]. Thus it can be concluded that the excitations that give rise to this feature involve cooperative motion that extends at least over several $\mathrm{nm}$ and that, in order to finally find the answer concerning the nature of the peak, large systems $\left[\mathrm{O}\left(10^{4}\right)\right.$ ions $]$ have to be analyzed so that the mentioned finite size effects can be avoided.

A further difference between the relaxation behavior of strong and fragile glass formers is concerned with the temperature dependence of the height of the plateau in time correlation functions. This effect can be studied best if one plots the correlation functions versus the rescaled time $t / \tau(T)$, where $\tau(T)$ is the $\alpha$-relaxation time at temperature $T$. This time can, e.g., be defined as the time it takes a correlation function to decay to $e^{-1}$ of its initial value. (Another possibility would be to define it as the area under the correlator.) In Fig. 6 we show the so obtained figures for the same correlators shown in Fig. 5. For the fragile glass former we find that, at low temperatures, this scaling leads to a master curve which extends throughout the whole $\alpha$-regime, i.e. that part of the relaxation in which the correlators fall below the plateau. Such a master curve has been found also in other simulations [25 27,66, 71] and in experiments [12] and its existence is one of the important predictions of MCT. The existence of this master curve is by no means trivial, as can be recognized from the corresponding plot for silica (Fig. 6 6b) since for this systems the scaled curve do, in the vicinity of the plateau, not fall at all onto a master curve. A detailed analysis of the individual curves shows, however, that, in the $\beta$-relaxation regime, the shape of the curves is indeed independent of temperature [77,78]. Thus the only reason why they do not fall onto a master curve is the presence of the boson-peak at short times which leads to a temperature dependent height of the plateau. Also in experiments it has been found that 
in strong glass formers the boson-peak dominates the time dependence of the correlation function in the time range where the correlators approach the plateau [10]. Most of these findings stem, however, from experiments in which the system is probed in the frequency domain (such as light scattering). Therefore such experiments, if done at frequencies $\omega>0$, will in general not notice whether or not the height of the plateau depends on temperature, since such a dependency will affect only the intensity of the signal at frequency zero. Thus the results from the computer simulation do indeed give new insight into the dynamics of strong glass formers.

Above we have shown that for the case of silica the power-law predicted by MCT for the temperature dependence of $D$ can be observed only for a relatively small range of the diffusion constant. From Fig. 6b one sees that, due to the strong influence of the bosonpeak in the $\beta$-relaxation regime, also the predicted master curve is not observed. Therefore one might be tempted to argue that MCT is not a very useful description of the dynamics of $\mathrm{SiO}_{2}$. This point of view is, however, far too pessimistic, since there are predictions of the theory which are valid also in the presence of the mentioned hopping processes, such as, e.g., the so-called factorization property, which states that in the beta-relaxation regime any time correlation function $\Phi(t)$ can be written as $\Phi(t)=f+h G(t)$, where $G(t)$ is a system universal function and $f$ and $h$ will depend on the correlation function $\Phi$. It has been shown [77,78 that for the case of silica this factorization property holds very well, thus showing that MCT is able to make relevant (and correct) predictions on the dynamics for this (strong) type of glass former as well.

Having now some understanding of the temperature dependence of the relaxation dynamics of the Lennard-Jones and the silica system we can compare it with that of other glass-formers. Sciortino et al. have done extensive simulations of the dynamics of supercooled water [83], a system which shares many structural and thermodynamical properties with silica [3, 30,62,85]. These simulations have shown that in the temperature range accessible to equilibrium simulations, the diffusion constant shows a power-law dependence [83], a result that is supported also by experiments [3, 86]. Thus, although the system is structurally much more similar to the strong glass former silica, it behaves dynamically like a fragile glass former. From our findings for silica we therefore conclude that the strong hopping processes found in silica are less pronounced in water than in silica (which might, however, also have to do with the fact that the water simulations have been done with $\mathrm{H}_{2} \mathrm{O}$ molecules that could not dissociate).

For the early $\beta$-relaxation regime it is found [83] that also water shows a small dip, as we showed it to be present in silica (see Fig. 6]b). However, contrary to the case of silica, this dip does not destroy the master curve in the late $\beta$-relaxation regime when the correlators are plotted versus $t / \tau(T)$. Thus the dynamics of water seems to behave in certain aspects like a fragile glass former and in other aspects like a strong one. This duality is most likely due to the network structure since the simple atomic and molecular liquids, that do not have the tendency to form a network, have been found to show essentially the same dynamics as the Lennard-Jones system discussed here [25, 27,66, 71, 87]. Also the dynamics of polymeric glass formers is in many aspects quite similar to the one of simple liquids 26. However, because of the length of the molecules these systems do also show interesting dynamic effects which are not present in simple liquids, such as the motion of side chains or reptative movements. For a more thorough discussion of these issues we refer the reader to the review articles by 
Clarke [88] and Paul and Baschnagel [89].

The results presented so far are mainly useful to understand the slowing down of the relaxation dynamics on a qualitative level. However, it is also possible to use computer simulations to study this relaxation dynamics on a quantitative level and thus to test predictions of theories that attempt to describe this dynamics. In the following we will therefore briefly describe a few simulations which have been done to test the validity of such theories.

As already mentioned in Section $\mathbb{\mathbb { 1 }}$, two very prominent theories are the ones of Gibbs and DiMarzio [20], which is very popular in the community of polymer scientists, and the so-called mode-coupling theory which seems to be applicable for a very large variety of glassformers [11]. The basic idea of theses two theories is very different. In the Gibbs-DiMarzio approach one starts from polymers which are placed on a lattice. By counting the number of ways the polymers of the melt can be placed on the lattice, Gibbs and DiMarzio found that there exists a critical packing fraction above which this configurational entropy goes to zero in a continous way. From this they concluded the existence of a second order phase transition at this point, which is acompanied by the usual critical slowing down of the dynamics. This slowing down then gives rise to the glass transition. It has been found that this theory is able, e.g., to rationalize the dependence of the glass transition temperature on the length of the polymers, on the concentration of plastisizer, and other relevant quantities [90]. It was therefore quite surprising when a few years ago a simulation of Wolfgardt et al. showed that the observed slowing down of the relaxation dynamics of a dense polymer melt is not due to the fact that the configurational entropy of the system becomes zero [91]. This can be seen in Fig. 0 in which the entropy is plotted versus the inverse temperature. The open symbols correspond to the entropy as given by the (approximate) expression proposed by Gibbs and DiMarzio and one sees that this prediction gives a zero value of the entropy at a finite temperature. (It has to be emphasized that the quantities entering the Gibbs-DiMarzio expression have been calculated from the simulation as well and thus no approximation on that level is made.) The filled symbols are the real values of the entropy of the system which have been measured by thermodynamic integration. From the figure it becomes clear that this real entropy does not go to zero in the temperature range in which the GibbsDiMarzio expression becomes zero, that, however, it shows a noticeable decrease in this temperature range. Thus one has to conclude that the success of the Gibbs-DiMarzio theory relies probably on the fact that the real entropy decreases significantly in the vicinity of the critical temperature, that the theory gives a quite realistic description of this decrease on the qualitative level, and that most experiments measure only quantities which are related to the various derivatives of the entropy.

The second theory that we will discuss here to some extend, the mode-coupling theory, has a very different explanation for the slowing down of the system upon cooling. From the theory of the dynamics of simple liquids in the vicinity of the triple point it is known that the equations of motion for density correlation functions have nonlinear terms which are needed to describe back-flow effects and cage effects [92 and MCT is such a set of equations of motion. It is found that with decreasing temperature the nonlinear terms become stronger and will lead to a dynamical feedback effect that slows down the relaxation of the density correlators. Within a certain approximation to these equations, leading to what is known as the "ideal MCT", this feedback mechanism becomes so strong that at a certain temperature $T_{c}$ the correlation functions do not decay to zero anymore, i.e. the system has undergone 
a glass transition. Using this $T_{c}$ as a reference temperature, the theory makes detailed predictions regarding the time and temperature dependence of correlation functions, such as the intermediate scattering function discussed above.

One of the important aspects of the theory is that the equations of motion for the density correlators depend only on static quantities, such as the structure factor. Since such quantities can be obtained quite easily with high precision from experiments or simulations, it is thus possible to measure the static quantities, solve the MCT equations and compare the so obtained theoretical curves for the time dependent correlation functions with the one measured in the experiment or in the computer simulation. Therefore very stringent tests of the theory become possible on a qualitative as well as quantitative level.

The outcome of such a test has recently been reported by Nauroth et al. [93]. In that work the MCT equations for the binary Lennard-Jones system discussed above have been solved numerically and their solutions at long times compared with the results from simulations. Among the quantities investigated were the wave-vector dependence of the height of the plateau in the intermediate scattering function (see Fig. 5) at the critical temperature $T_{c}$, a quantity which is also called the Edwards-Anderson, or nonergodicity parameter $f_{c}(q)$.

In Fig. 8 we show $f_{c}(q)$ as predicted by MCT (solid curves) and the one from the computer simulation (open symbols). The Gaussian shaped curve is the nonergodicity parameter for the incoherent intermediate scattering function $F_{s}$ of the $A$ particles [Eq. (3)] and the oscillatory curves are for the coherent intermediate scattering function for the $A-A$ correlation [21. We emphasize that for the calculation of the theoretical curves no fit parameter of any kind was used. The only input was the temperature dependence of the partial structure factors, which were determined from the simulation. From the figure we recognize that the theory gives an excellent description of the wave-vector dependence of $f_{c}$ and we thus conclude that the theory is indeed able to make precise quantitative predictions on the dynamics of supercooled simple liquids.

Also included in the figure are the results of a computer simulation of the mentioned binary Lennard-Jones system, but instead of the Newtonian dynamics used in Ref. [72] a Brownian-like (stochastic) dynamics was used [94. In this type of dynamics the equations of motion of the particles are given by

$$
m \ddot{\mathbf{r}}_{j}+\sum_{i \neq j} \frac{\partial V\left(\left|\mathbf{r}_{i}-\mathbf{r}_{j}\right|\right)}{\partial \mathbf{r}_{j}}+\zeta \dot{\mathbf{r}}_{j}+\eta_{j}(t)=0
$$

where $\zeta$ is a friction constant whose value is related to the amplitude of the white noise $\eta_{j}(t)$ by the fluctuation dissipation theorem, i.e. $\left\langle\eta_{j}(t) \eta_{i}\left(t^{\prime}\right)\right\rangle=6 k_{B} T \delta\left(t-t^{\prime}\right) \delta_{j i}$. There are two reasons for studying a system with such a stochastic dynamics. Firstly the system with these stochastic forces will probably have a dynamics which is quite similar to the one of a colloidal particle in a suspension, a model system for which beautiful light scattering experiments have been performed to study the glass transition [12]. Thus it is interesting to see whether the relaxation dynamics of the Lennard-Jones system is indeed very similar to the one found in these experiments. The second reason is related to a prediction of MCT which states that at long times the relaxation dynamics is independent of the microscopic dynamics. Thus we can compare the relaxation behavior of the system with the stochastic dynamics with the one of the Newtonian dynamics and see in what aspects the two differ. Work in this direction has already been done by Löwen et al. [95]. These authors made a simulation 
of a supercooled polydisperse mixture of particles interacting with a Yukawa potential and compared the relaxation dynamics of this system with a Newtonian dynamics to the one with a Brownian dynamics. It was found that in the $\beta$-relaxation regime the dynamics depends on the microscopic dynamics, that, however, the $\alpha$-relaxation was independent of it. Qualitatively similar results have been found by Gleim et al. in the mentioned simulation of the Lennard-Jones system 94,96. In that work it was shown that at low temperatures the whole relaxation dynamics is independent of the microscopic dynamics, if one leaves aside the relaxation at very short (microscopic) times. An example for this finding is shown in Fig. 8, were we have also included the wave-vector dependence of the nonergodicity parameter for the system with the stochastic dynamics (filled symbols). We see that this $q$-dependence is essentially independent of the microscopic dynamics, thus confirming this prediction of the theory. Furthermore we also recognize that essentially for all wave-vectors the curves for the stochastic dynamics agree even better with the theoretical ones than the curves for the Newtonian dynamics do. The reason for this might be that MCT assumes that the time scale of the $\beta$-relaxation, at which the height of the plateau is measured, is separated well from the one of the microscopic relaxation. Since in the stochastic dynamics the phonons are strongly damped it can be expected that effectively this separation is larger in the case of the stochastic dynamics than for the case of the Newtonian dynamics, where no damping is present, and that therefore the assumption of the theory is better fulfilled.

\section{B. Dynamics below the glass transition temperature}

In the previous subsection we have discussed the equilibrium relaxation dynamics of glassforming liquids in their supercooled state, i.e. at temperatures above the glass transition temperature $T_{g}$. Since we have seen that with decreasing temperature the relaxation time $\tau$ increases quickly, we expect that there will exist a temperature $T_{g}$ at which $\tau$ will exceed the time scale of the experiment or simulation and hence it will no longer be possible to equilibrate the system. One might guess that at temperatures below $T_{g}$ the system is essentially frozen, i.e. that its dynamics is similar to the vibrational dynamics of a solid. To a certain extend this view is certainly correct and solid state concepts, like, e.g., the density of states, have successfully been applied to describe the dynamics of disordered systems or to compute their specific heat 97. However, as we will show in this section, even at low temperatures the dynamics of disordered systems is not purely of vibrational type but has also a very interesting relaxational component. This slow relaxation is known as "aging" and leads to a slow time dependence of various material properties, such as brittleness, density, etc. The importance of such aging phenomena has been realized for quite a long time 98 (and they have been described by means of phenomenological theories) but it is only in recent years that sound theoretical concepts have been developed in order to describe this type of dynamics [99 102]. Apart from extensive investigations on polymeric systems 98, aging phenomena have so far mainly been investigated in spin glasses by means of experiments and computer simulations [16,99, 103, 104]. Only very recently experiments and computer simulations of structural glasses and other disordered systems have been made in order to investigate these phenomena [70,105]111] so that the validity of the various theoretical approaches can be tested. The results of these investigations do not yet allow to decide which one of the theoretical pictures proposed ("droplet model", "trap model", mean- 
field) 99 102,106 is appropriate to describe the aging dynamics of structural glasses but it can be expected that further studies will ultimatively rule out certain scenarios. Therefore the goal of the following presentation of the results of such investigations is not to advocate any particular theory but rather to familiarize the reader with the occurring effects and to show how computer simulations might help to decide which theoretical picture is appropriate to describe this aging dynamics.

In order to study aging effects it is of course necessary to first drive the system out of equilibrium. In structural glasses this can be done, e.g, by decreasing the temperature below the glass transition temperature or by compressing the system beyond a "critical" density. If the out of equilibrium situation is generated by a temperature jump, one proceeds as follows. The system is first equilibrated at an initial temperature $T_{i}$. At time $t=0$ the system is quenched to a temperature $T_{f}$ which is significantly lower than the glass transition temperature $T_{g}$, where $T_{g}$ is given by the time scale of the experiment. After the quench the system is allowed to evolve for a waiting time $t_{w}$, after which the measurements start. The relaxation of the system is now studied as a function of $t_{w}$ and $\tau$, the time elapsed since the start of the measurement, i.e. since $t=t_{w}$. In a recent computer simulation Kob and Barrat studied the relaxation dynamics of the binary Lennard-Jones system discussed above after such a quench [108]. In Fig. 9 we show the time dependence of $e_{p o t}$, the potential energy of the system, after such a quench to $T_{f}=0.4$ for three different initial temperatures $T_{i}=5.0,0.8$ and 0.466 . Note that the glass transition temperature of the system for very long simulations $\left(\mathrm{O}\left(10^{8}\right)\right.$ time steps) is around $T_{c}=0.435$ [72]. One recognizes that, after a relatively fast decay, the potential energy for the two larger values of $T_{i}$ is almost constant and also the curve for the lowest $T_{i}$ depends only weakly on time. A more careful analysis of this time dependence shows that it is approximated well by a power-law with a small exponent around 0.14, or by a logarithmic time dependence. Since this dependence is so weak one therefore might erroneously conclude that at the end of the run the system has equilibrated, or is at least quite close to equilibrium. We know, however, that this is clearly not the case, since we are at a temperature at which the relaxation time of the system exceeds by several orders of magnitude the time scale of the simulation. Hence this should be taken as a warning in using a one-time quantity as a probe of whether or not the system has been equilibrated or not. (By "one-time quantity" we mean an observable which in equilibrium is a constant, such as the energy, pressure or density.) Thus we conclude that the investigation of such quantities is not very well suited to study aging phenomena, a result in agreement with simulations by Andrejew and Baschnagel of aging effects in a polymer melt [110].

Much more sensitive quantities to study the aging dynamics are "two-time" correlation functions, such as the generalization of the intermediate scattering function $F_{s}(q, t)$ [Eq. (3)] to a non-equilibrium situation. We recall that by definition of equilibrium, a time correlation function depends only on time differences, i.e.

$$
F_{s}(q, \tau)=\frac{1}{N} \sum_{j=1}^{N}\left\langle\exp \left(i \mathbf{q} \cdot\left[\mathbf{r}_{j}(\tau)-\mathbf{r}_{j}(0)\right]\right\rangle=\frac{1}{N} \sum_{j=1}^{N}\left\langle\exp \left(i \mathbf{q} \cdot\left[\mathbf{r}_{j}\left(t_{w}+\tau\right)-\mathbf{r}_{j}\left(t_{w}\right)\right]\right\rangle\right.\right.
$$

Since the quench breaks the time translation invariance of the system, the second equality no longer holds and we therefore define the out of equilibrium time correlation function

$$
C_{q}\left(t_{w}+\tau, t_{w}\right)=\frac{1}{N} \sum_{j=1}^{N}\left\langle\exp \left(i \mathbf{q} \cdot\left[\mathbf{r}_{j}\left(t_{w}+\tau\right)-\mathbf{r}_{j}\left(t_{w}\right)\right]\right\rangle\right.
$$


Here $\langle$.$\rangle stands for the average over the temperature before the quench. The time dependence$ of $C_{q}\left(t_{w}+\tau, t_{w}\right)$ for different waiting times is shown in Fig. 10. We see that this two-time correlation function shows a very strong dependence on the waiting time and is therefore well suited to study the aging dynamics. For short $t_{w}$ the function decays very quickly, since the typical configuration of the particles at $T_{f}$ is very different from the one at $T_{i}$. Therefore the system is subject to a large driving force which will move it towards a part of phase space which is more typical for the temperature $T_{f}$ and thus the system will quickly decorrelate from the initial configuration. If the waiting time is increased, the driving force is smaller and at short and intermediate times $\tau$ the system will explore only that part of phase space which corresponds to the vibrational motion of the particles in their cages. This is the reason why the correlation functions show a plateau at intermediate times and look qualitatively quite similar to the ones in equilibrium (cf. Fig. 5a). Only for larger times $\tau$ does the correlation function decay to zero and the time at which this final decay is observed increases with $t_{w}$. Although the off-equilibrium relaxation curves $C_{q}\left(t_{w}+\tau, t_{w}\right)$ for large $t_{w}$ look qualitatively similar to the ones in equilibrium [i.e. to $F_{s}(q, t)$ ] a detailed analysis shows that there are important differences. E.g. it is well known that at long times the equilibrium relaxation curves of glass forming liquids can be fitted very well by a Kohlrausch-Williams-Watts function, $A \exp \left(-\left(t / \tau_{K W W}\right)^{\beta}\right)$, and this is also the case for the present Lennard-Jones system [72]. For the out of equilibrium function it is, however, found that the relaxation at long times is given by a power law with an exponent around 0.4 [111] a result which is in qualitative agreement with results of simulations of spin glasses [16,104. Hence we conclude that despite the apparent similarity of the curves for equilibrium and out of equilibrium relaxation, the two types of functions differ significantly. Therefore it is not appropriate to use the latter ones as an approximation for the former ones, as it is sometimes done when time correlation functions for temperatures above and below $T_{g}$ (as determined from the simulation) are mixed together in the analysis of the data.

In the discussion of Fig. 9 we concluded that a one-time quantity is not a good indicator for whether or not the system has reached equilibrium. Often it is argued that the decay of a time correlation function, or seeing a diffusive (i.e. linear) time dependence of the mean squared displacement within the time span of the simulation, is a sufficient condition for having reached equilibrium. From Fig. 10 one recognizes however, that this is not the case at all, since the correlation functions decay also in the non-equilibrium situation, a result which was also demonstrated nicely by Baschnagel [112], and in a recent simulation of a soft sphere system Parisi demonstrated that a linear dependence of the MSD does not imply at all that one has reached equilibrium [70].

One of the interesting results of the theories of aging concerns the violation of the fluctuation-dissipation theorem (FDT) 99, 101 which in equilibrium relates $R_{A}(t)$, the response of an observable $A$ to its conjugate field, to the time autocorrelation function $C_{A}(t)=\langle A(t) A(0)\rangle$, i.e. $R_{A}(t)=-\left(k_{B} T\right)^{-1} \partial C_{A}(t) / \partial t$. For the out of equilibrium case this relation is no longer valid and it is generalized to

$$
R_{A}\left(t^{\prime}, t\right)=\frac{1}{k_{B} T} X_{A}\left(t^{\prime}, t\right) \frac{\partial C_{A}\left(t^{\prime}, t\right)}{\partial t},
$$

where $t^{\prime} \geq t$ and the quantity $X_{A}\left(t^{\prime}, t\right) \leq 1$ is defined by this equation. From this relation it becomes clear that the quantity $T / X_{A}\left(t^{\prime}, t\right)$ can be viewed as the effective temperature for 
which the FTD holds 113] (see also Ref. [101). Note that in the field of glass science the concept of a "fictive" temperature has been introduced long ago by Tool and Eichlin [114] but, to our knowledge, has never been based on a solid statistical mechanics foundation. In contrast to this the definition of such a temperature via Eq. (7) does not have this drawback and permits to measure this temperature in experiments or simulations. From the equation it also becomes immediately obvious that the ratio $T / X_{A}\left(t^{\prime}, t\right)$ will in general depend on time and on the observable considered.

If the observable of interest is the coherent intermediate scattering function $C_{q}\left(t_{w}+\tau, t_{w}\right)$ one can measure the response $R\left(t_{w}+\tau, t_{w}\right)$ (in principle) as follows $t$. After the waiting time $t_{w}$, a space dependent sinusoidal field with wave-vector $q$, which couples to the particle density and has an amplitude $V_{0}$, is turned on and the resulting change in the density distribution for wave-vector $q$ is measured. After a measuring time $\tau$ one therefore obtains the integrated response $M\left(t_{w}+\tau, t_{w}\right)$ given by

$$
V_{0} M\left(t_{w}+\tau, t_{w}\right)=V_{0} \int_{t_{w}}^{t_{w}+\tau} R\left(t_{w}+\tau, t\right) d t .
$$

For large values of $\tau$ and $t_{w}$ it is expected that the FDT-violation factor $X\left(t_{w}+\tau, t_{w}\right)$ in Eq. (7) becomes a function of $C\left(t_{w}+\tau, t_{w}\right)$ only, i.e. $X\left(t_{w}+\tau, t_{w}\right)=x\left(C\left(t_{w}+\tau, t_{w}\right)\right)$, where $x$ is a function of one variable [99]. From this relation and Eq. (8) one thus derives

$$
M\left(C_{q}\right)=\frac{1}{k_{B} T} \int_{C_{q}}^{1} x(c) d c,
$$

where we used the fact that $C_{q}\left(t_{w}+\tau, t_{w}\right)=1$ for $\tau=0$. This equation thus says that a parametric plot of $k_{B} T M$ versus $C_{q}$, with time $\tau$ as a parameter, will give us information on the function $x(c)$ and hence on the FDT-violation factor $X\left(t_{w}+\tau, t_{w}\right)$.

In Fig. 11 we show such a parametric plot for the same correlation function shown in Fig. 10 and the corresponding integrated response $M$. The values of $t_{w}$ and $T_{f}$ are 1000 and 0.4 , respectively. We see that for short times $\tau$, corresponding to large values of $C_{q}$, the data points can be approximated well by a straight line with a slope around -1 (see figure). This means that the FDT-violation factor $X$ is 1, i.e. that the FDT holds and the system behaves like in equilibrium. However, for larger times (corresponding to smaller values of $C_{q}$ ) the FDT is violated since the data points do not fall anymore on a straight line with slope -1 . What is observed instead is a straight line with a slope around -0.62 . This means that in this time regime $X\left(t_{w}+\tau, t_{w}\right)$ is constant and has a value around 0.62 , which corresponds to an effective temperature of $0.4 / 0.62 \approx 0.64$. Qualitatively similar results have been found in the seminal work of Parisi in which the violation of the FDT has been investigated for a soft sphere system [70]. In that simulation the observable of interest was the mean squared displacement and it was shown that the FDT violation factor $X$ shows a linear dependence on the final temperature $T_{f}$ of the quench, a result which has been confirmed also by Barrat and Kob [109].

Note that the fact that in the non-FDT region we find a straight line with a finite slope is not trivial at all, since certain theories of aging, such as domain growth [102], predict

\footnotetext{
${ }^{1}$ More details on this calculation can be found in Ref. 109.
} 
a straight line with slope zero and others a parabola-like dependence [99]. Hence we see that these type of measurements can indeed be used to collect evidence for or against a theoretical scenario.

\section{CONCLUSION}

In this review we have discussed some results of computer simulations of supercooled liquids. By now the literature on this topic is so vast that a comprehensive review is unfortunately no longer possible and thus we have focussed on only a few topics, namely on the (metastable) equilibrium dynamics above $T_{g}$ for a strong and fragile glass former and the non-equilibrium dynamics below $T_{g}$. Whereas the investigation of the former type of question is today still dominated by real experimentalist, the latter seems to have been studied in much more detail by means of computer simulations, since for the moment they are better adapted to investigate such problems. We hope however that in the near future this situation changes, since such investigations allow to learn more about the structure of the phase space of the system. (We mention that in the past some properties of phase space have been investigated by determining the inherent structure of liquids and studying normal modes [115.) If the structure of this phase space is understood well, e.g. whether or not it is organized in a hierarchical way, it might become possible to understand the connection between systems in which the disorder is quenched (such as spin glasses) and systems with self generated disorder (e.g. structural glasses).

Other types of questions in which computer simulations are probably very valuable, are to investigate the equilibrium dynamics of supercooled liquids in the region between the MCT temperature $T_{c}$ and the experimental glass transition $T_{g}$ on a microscopic level. Although for fragile glass formers such simulations are currently not quite feasible, they will be possible in a few years. Since at the moment there is no complete theory for the dynamics of liquids in this temperature range and real experiments rarely allow to probe the system on the microscopic level in sufficient detail, such simulations will be an excellent tool to gain insight into this question and thus possibly serve as a guide to the development of reliable theories.

Apart from these types of simulations which are motivated mainly by the wish to have a sound theoretical understanding of glassy materials, there are of course also those simulations which are done to calculate properties of specific materials. For example it is possible to predict the temperature dependence of the specific heat of silica, for temperatures above $100 \mathrm{~K}$ up to $T_{g}$, to within a few percent of the experimental values [97, or the dynamic structure factor with quite high accuracy [42, 77, ,78]. It can be expected that in a few years the quality of the available potentials will increase even further and that soon also potentials of a bit more exotic materials (such as multicomponent systems) will be determined. This will then in turn open the door to many types of simulations related more closely to materials science and thus allow to use all the know-how and insight gained in the investigation of less complex models also in technological more relevant materials.

Acknowledgements: I thank H. C. Andersen, C. A. Angell, J.-L. Barrat, K. Binder, T. Gleim, J. Horbach, M. Nauroth, and K. Vollmayr with whom I had the pleasure to collaborate on some of the work that was presented here, and W. Paul for useful comments on the manuscript. I am also deeply grateful to all the people who have taught me most of the 
things I know on this subject and whose shear number regretfully prevents me to list them here by name. Part of this work was supported by the Deutsche Forschungsgemeinschaft under SFB 262, the Pole Scientifique de Modélisation Numérique at the ENS-Lyon and the computing centers HLRZ in Jülich and RUS in Stuttgart. 


\section{REFERENCES}

[1] See, e.g., R.W. Douglas and S. Frank, A History of Glassmaking (G.T. Foulis, Henley on Thames, 1972).

[2] R. Zallen, The Physics of Amorphous Materials (Wiley, New York, 1983); J. Jäckle, Rep. Prog. Phys. 49, 171 (1986); D. R. Uhlmann and N. J. Kreidl (Eds.), Vol. 3 of Glass Science and Technology: Viscosity and Relaxation, (Academic Press, Orlando, 1986); J. Zarzycki (Ed.), Vol. 9 of Materials Science and Technology, (VHC, Weinheim, 1991); A. Feltz, Amorphous Inorganic Materials and Glasses (VCH, Weinheim, 1993); U. Mohanty, Adv. Chem. Phys. 89, 89 (1995); Proceedings of 3rd International Discussion Meeting on Relaxation in Complex Systems Ed.: K. L. Ngai, J. Non-Cryst. Solids 235238 (1998).

[3] P. G. Debenedetti, Metastable Liquids (Princeton University Press, Princeton, 1997).

[4] K. Binder and A. P. Young, Rev. Mod. Phys. 58, 801 (1986).

[5] N. Metropolis, A. W. Rosenbluth, M. N. Rosenbluth, A. H. Teller, and E. Teller, J. Chem. Phys. 21, 1087 (1953); B. J. Alder and T. E. Wainwright, J. Chem. Phys. 27, 1208 (1957).

[6] M. P. Allen and D. J. Tildesley, Computer Simulation of Liquids (Oxford University Press, New York, 1990); D. Frenkel and B. Smit, Understanding Molecular Simulation From Algorithms to Applications (Academic Press, San Diego, 1996); K. Binder and G. Ciccotti (Eds.), Monte Carlo and Molecular Dynamics of Condensed Matter Systems (Italian Physical Society, Bolognia, 1996); K. Binder and D. W. Heermann, Monte Carlo Simulation in Statistical Physics (Springer, Berlin, 1997).

[7] C. A. Angell, J. H. R. Clarke, and L. V. Woodcock, Adv. Chem. Phys. 48, 397 (1981); J.-L. Barrat and M. L. Klein, Ann. Rev. Phys. Chem. 42, 23 (1991).

[8] P. H. Poole, P. F. McMillan, and G. H. Wolf, p. 563 in Structure, Dynamics and Properties of Silicate Melts, Eds.: J. F. Stebbins, P. F. McMillan, D. B. Dingwell, (Mineralogical Society of America, Washington, 1995); K. Binder and W. Kob, to appear in Analysis of Composition and Structure of Glass, and Glass Ceramics Eds.: H. Bach and D. Krause (Springer, Berlin, 1998); W. Kob and K. Binder, to appear in Analysis of Composition and Structure of Glass, and Glass Ceramics Eds.: H. Bach and D. Krause (Springer, Berlin, 1998).

[9] J. Wuttke, J. Hernandez, G. Li, G. Coddens, H. Z. Cummins, F. Fujara, W. Petry, and H. Sillescu, Phys. Rev. Lett. 72, 3052 (1994).

[10] E. Rössler, A.P. Sokolov, A. Kisliuk, and D. Quitmann, Phys. Rev. B 49, 14967 (1994).

[11] W. Götze, p. 287 in Liquids, Freezing and the Glass Transition Eds.: J. P. Hansen, D. Levesque and J. Zinn-Justin, Les Houches. Session LI, 1989, (North-Holland, Amsterdam, 1991); W. Götze and L. Sjögren, Rep. Prog. Phys. 55, 241 (1992); W. Kob, p. 28 in Experimental and Theoretical Approaches to Supercooled Liquids: Advances and Novel Applications Eds.: J. Fourkas, D. Kivelson, U. Mohanty, and K. Nelson (ACS Books, Washington, 1997).

[12] W. van Megen and P.N. Pusey, Phys. Rev. A 43, 5429 (1991); E. Bartsch, M. Antonietti, W. Schupp, and H. Sillescu, J. Chem. Phys. 97, 3950 (1992); W. van Megen and S.M. Underwood, Phys. Rev. E 49, 4206 (1994).

[13] G. H. Fredrickson and H. C. Andersen, J. Chem. Phys. 83, 5822 (1985); G. H. Fredrickson and S. A. Brawer, J. Chem. Phys. 84, 3351 (1986); T. A. Weber, G. H. Fredrickson, 
and F. H. Stillinger, Phys. Rev. B 34, 7641 (1986); W. Ertel, K. Froböse, and J. Jäckle, J. Chem. Phys. 88, 5027 (1988); K. Froböse, J. Stat. Phys. 55, 1285 (1989); J. Jäckle, K. Froböse, and D. Knödler, J. Stat. Phys. 63, 249 (1991); W. Kob and H. C. Andersen, Phys. Rev. E 47, 3281 (1993); ibid. 48, 4364 (1993).

[14] R. Brüning and K. Samwer, Phys. Rev. B, 46, 11318 (1992).

[15] C. A. Angell, in Relaxation in Complex Systems, Eds.: K. L. Ngai and G. B. Wright (US Dept. Commerce, Springfield, 1985).

[16] H. Rieger, p. 295 in Vol. II of Annual Reviews of Computational Physics, Ed.: D. Stauffer (World Scientific, Singapore, 1995).

[17] A. P. Young (Ed.), Spin Glasses and Random Fields (World Scientific, Singapore, 1998).

[18] D. Sherrington, to appear in Proceedings of 1997 Canberra Summer School on "The Physics of Novel Materials", preprint cond-mat/9806289.

[19] W. Kob, p. 1 in Vol. III of Annual Reviews of Computational Physics, Ed.: D. Stauffer (World Scientific, Singapore, 1995).

[20] J. H. Gibbs and E. A. DiMarzio, J. Chem. Phys. 28, 373 (1958); E. A. DiMarzio and J. H. Gibbs, J. Chem. Phys. 28, 807 (1958).

[21] J.-P. Hansen and I. R. McDonald, Theory of Simple Liquids (Academic, London, 1986); U. Balucani and M. Zoppi, Dynamics of the Liquid State (Oxford University Press, Oxford, 1994).

[22] F. Ritort, Phys. Rev. Lett. 75, 1190 (1995); S. Franz and F. Ritort, Europhys. Lett. 31, 507 (1995); A. Prados, J.J. Brey, and B. Sánchez-Ray, Phys. Rev. B 55, 6343 (1997).

[23] J. Kurchan, L. Peliti, and M. Sellitto, Europhys. Lett. 39, 365 (1997).

[24] G. F. Signorini, J.-L. Barrat, and M. L. Klein, J. Chem. Phys. 92, 1294 (1990); P. Sindzingre and M. L. Klein, J. Chem. Phys. 96, 4681 (1992); S. R. Kudchadkar and J. M. Wiest, J. Chem. Phys. 103, 8566 (1995); J. Baschnagel, K. Binder, and H. P. Wittmann, J. Phys.: Condens. Matter 5, 1597 (1993); B. Lobe and J. Baschnagel, J. Chem. Phys. 101, 1616 (1994); C. Renner, H. Löwen, and J.-L. Barrat, Phys. Rev. E 52, 5091 (1995).

[25] L. J. Lewis and G. Wahnström, Solid State Comm. 86, 295 (1993); Phys. Rev E 50, 3865 (1994).

[26] C. Bennemann, W. Paul, K. Binder, and B. Dünweg, Phys. Rev. E 57, 843 (1998).

[27] S. Kämmerer, W. Kob, and R. Schilling, Phys. Rev. E 56, 5450 (1997); ibid. 58, 2131 (1998); ibid. 58, 2141 (1998)

[28] J. R. Rustad, D. A. Yuen, and F. R. Spera, Phys. Rev. B 44, 2108 (1991).

[29] W. Jin, R. K. Kalia, P. Vashishta, and J. P. Rino, Phys. Rev. Lett. 71, 3146 (1993).

[30] P. H. Poole, M. Hemmati, and C. A. Angell, Phys. Rev. Lett. 79, 2281 (1997).

[31] J. Badro, P. Gillet, and J.-L. Barrat, Europhys. Lett. 42, 643 (1998).

[32] D. L. Lacks, Phys. Rev. Lett. 80, 5385 (1998).

[33] J. Baschnagel and K. Binder, Macromolecules, 28, 6808 (1995).

[34] T. Fehr and H. Löwen, Phys. Rev. E 52, 4016 (1995).

[35] F. Sciortino, P. H. Poole, U. Essmann, and H. E. Stanley, Phys. Rev. E 55, 727 (1997); H. Tanaka, Phys. Rev. Lett. 80, 113 (1998).

[36] J. Badro, D. M. Teter, R. T. Downs, P. Gillet, R. J. Hemley, and J.-L. Barrat, Phys. 
Rev. B 56, 5797 (1997).

[37] J. Sarnthein, A. Pasquarello, and R. Car, Phys. Rev. Lett. 74, 4682 (1995); A. Pasquarello, J. Sarnthein, and R. Car, Phys. Rev. B 57, 14133 (1998).

[38] A. Pasquarello and R. Car, Phys. Rev. Lett. 80, 5145 (1998).

[39] W. C. Swope, and H. C. Andersen, Phys. Rev. B 41, 7042 (1990); P. Vashishta, R. K. Kalia, W. Li, A. Nakano, A. Omeltchenko, K. Tsuruta, J. H. Wang, and I. Ebbsjö, Current Opinion Solid State and Materials Science 1, 853 (1996).

[40] J. Horbach, W. Kob, K. Binder, and C. A. Angell, Phys. Rev. E. 54, R5897 (1996).

[41] U. Buchenau, M. Prager, N. Nücker, A. J. Dianoux, N. Ahmad, and W. A. Phillips, Phys. Rev. B. 34, 5665 (1986); P. Benassi, M. Krisch, C. Masciovecchio, V. Mazzacurati, G. Monaco, G. Ruocco, F. Sette, and R. Verbeni, Phys. Rev. Lett. 77, 3835 (1996); M. Foret, E. Courtens, R. Vacher, and J.-B. Suck, Phys. Rev. Lett. 77, 3831 (1996); W. Schirmacher, G. Diezemann, and C. Ganter, Phys. Rev. Lett. 81, 136 (1998); A. Wischnewski, U. Buchenau, A. J. Dianoux, W. A. Kamitakahara, and J. L. Zarestky, Phys. Rev. B 57, 2663 (1998).

[42] S. N. Taraskin and S. R. Elliott, Europhys. Lett. 39, 37 (1997); Phys. Rev. B 56, 8605 (1997).

[43] See., e.g., A. D. Sokal, p. 47 in Monte Carlo and Molecular Dynamics Simulations in Polymer Science Ed.: K. Binder (Oxford University Press, New York, 1995).

[44] C. Dress and W. Krauth, J. Phys. A: Math. Gen. 28, L597 (1995); W. Krauth and O. Pluchery, J. Phys A.: Math Gen. 27, L715 (1994); M. Achenbach, Diploma Thesis, University of Mainz (1996); G. T. Barkema and N. Mousseau, Phys. Rev. Lett. 77, 4358 (1996); F.-M. Dittes, Phys. Rev. Lett. 76, 4651 (1996); U. H. E. Hansmann and Y. Okamoto, Phys. Rev. E 56, 2228 (1997); G. A. Huber and J. A. McCammon, Phys. Rev. E 55, 4822 (1997); K. K. Bhattacharya and J. P. Sethna, Phys. Rev. E 57, 2553 (1998); J. P. K. Doye and D. J. Wales, Phys. Rev. Lett. 80, 1357 (1998); M. M. Steiner, P.-A. Genilloud, and J. W. Wilkins, Phys. Rev. B 57, 10236 (1998).

[45] K. Schmidt-Rohr and H.W. Spiess, Phys. Rev. Lett. 66, 3020 (1991); R. Böhmer, G. Hinze, G. Diezemann, B. Geil, and H. Sillescu, Europhys. Lett. 36, 55 (1995); A. Heuer, M. Wilhelm, H. Zimmermann and H.W. Spiess, Phys. Rev. Lett. 95, 2851 (1995); M.T. Cicerone, F. R. Blackburn and M. D. Ediger, J. Chem. Phys. 102, 471 (1995); M. T. Cicerone and M. D. Ediger, J. Chem. Phys. 104, 7210 (1996); H. Sillescu, J. Non-Cryst. Solids, (1998, in press).

[46] S. Butler and P. Harrowell, J. Chem. Phys. 95, 4466 (1991); A. Heuer and K. Okun, J. Chem. Phys. 106, 6176 (1997); W. Kob, C. Donati, S. J. Plimpton, S. C. Glotzer, and P. H. Poole, Phys. Rev. Lett. 79, 2827 (1997); C. Donati, J. F. Douglas, W. Kob, S. J. Plimpton, P. H. Poole, and S. C. Glotzer, Phys. Rev. Lett. 80, 2338 (1998); B. Doliva and A. Heuer, Phys. Rev. Lett. 80, 4915 (1998); S. C. Glotzer, N. Jan, T. Lookman, A. B. MacIsaac, and P. H. Poole, Phys. Rev. E 57, 7350 (1998); T. Muranaka and Y. Hiwatari, J. Phys. Soc. Japan 67, 1982 (1998); R. Yamamoto and A. Onuki, preprints cond-mat/9806207, cond-mat/9807180, and cond-mat/9807218.

[47] C. Dasgupta, A. V. Indrani, S. Ramaswamy, and M. K. Phani, Europhys. Lett. 15, 307 (1991); R. M. Ernst, S. R. Nagel, and G. S. Grest, Phys. Rev. B 43, 8070 (1991); K. Binder, J. Baschnagel, S. Böhmer, and W. Paul, Phil. Mag. B 77, 591 (1998).

[48] J. Habasaki, I. Okada, and Y. Hiwatari, Phys. Rev. E 52, 2681 (1995). 
[49] J. Horbach, W. Kob, and K. Binder, J. Non-Cryst. Solids, 235-238, xxxx (1998).

[50] R. Dell'Anna, G. Ruocco, M. Sampoli, and G. Viliani, Phys. Rev. Lett., 80, 1236 (1998).

[51] P. Maass, A. Bunde, and M. D. Ingram, Phys. Rev. Lett., 68, 3064 (1992); S. Balasubramanian and K. J. Rao, J. Phys. Chem. 97, 8835 (1993); J. Habasaki, I. Okada, and Y. Hiwatari, J. Non-Cryst. Solids, 208, 181 (1996).

[52] J. P. Rino, I. Ebbsjö, R. K. Kalia, A. Nakano, and P. Vashishta, Phys. Rev. B. 47, 3053 (1993).

[53] K. Vollmayr and W. Kob, Ber. Bunsenges. Phys. Chemie 100, 1399 (1996).

[54] K. Vollmayr, W. Kob, and K. Binder, Phys. Rev. B 54, 15808 (1996).

[55] A. Takada, C. R. A. Catlow, and G. D. Price, J. Phys.: Condens. Matter 7, 8659 (1995); ibid. 7, 8693 (1995).

[56] S. Tsuneyuki, M. Tsukada, H. Aoki, and Y. Matsui, Phys. Rev. Lett. 61, 869 (1988).

[57] B. W. H. van Beest, G. J. Kramer, and R. A. van Santen, Phys. Rev. Lett. 64, 1955 (1990).

[58] G. J. Kramer, A. J. M. de Man, and R. A. van Santen, J. Am. Chem. Soc. 113, 6435 (1991).

[59] X. Gonze, J.-C. Charlier, D. C. Allan, and M. P. Teter, Phys. Rev. B 50, 13035 (1994).

[60] M. Wilson and P. A. Madden, Phys. Rev. Lett. 72, 3033 (1994).

[61] M. Hemmati and C. A. Angell, in Physics meets Geology Eds.: H. Aoki and R. Hemley. (Cambridge Univ. Press, Cambridge, 1998).

[62] B. Vessal, M. Amini, D. Fincham, and C. R. A. Catlow, Phil. Mag. B 60, 753 (1989).

[63] R. G. Della Valle and E. Venuti, Chem. Phys. 179, 411 (1994); R. G. Della Valle and H. C. Andersen, J. Chem. Phys. 94, 5056 (1991); Y. Guissani and B. Guillot, J. Chem. Phys. 104, 7633 (1996).

[64] J. Horbach, W. Kob and K. Binder, Phil. Mag. B 77, 297 (1998).

[65] J. J. Ullo and S. Yip, Phys. Rev. Lett. 54, 1509 (1985); Phys. Rev. A 39, 5877 (1989); Chem. Phys. 149, 221 (1990).

[66] B. Bernu, Y. Hiwatari, and J.-P. Hansen, J. Phys. C 18, L371 (1985); B. Bernu, J.-P. Hansen, Y. Hiwatari, and G. Pastore, Phys. Rev. A 36, 4891 (1987); G. Pastore, B. Bernu, J.-P. Hansen, and Y. Hiwatari, Phys. Rev. A 38, 454 (1988); H. Miyagawa, Y. Hiwatari, B. Bernu, and J.-P. Hansen, J. Chem. Phys. 88, 3879 (1988); J.-N. Roux, J.-L. Barrat, and J.-P. Hansen, J. Phys.: Condens. Matter 1, 7171 (1989); J.-L. Barrat, J.-N. Roux, and J.-P. Hansen, Chem. Phys. 149, 197 (1990).

[67] H. Miyagawa and Y. Hiwatari, Phys. Rev. A 40, 6007 (1989); H. Miyagawa and Y. Hiwatari, Phys. Rev. A 44, 8278 (1991); Y. Hiwatari, J. Matsui, K. Uehara, T. Muranaka, H. Miyagawa, M. Takasu, and T. Odagaki, Physica A 204, 306 (1994); J. Matsui, T. Odagaki, and Y. Hiwatari, Phys. Rev. Lett. 73, 2452 (1994).

[68] M. M. Hurley and P. Harrowell, Phys. Rev. E 52, 1694 (1995); J. Chem. Phys. 105, 10521 (1996).

[69] G. Parisi, J. Phys. A: Math. Gen. 30, L765 (1997); J. Phys. A: Math. Gen. 30, 8523 (1997); B. Coluzzi and G. Parisi, J. Phys. A: Math. Gen. 31, 4349 (1998);

[70] G. Parisi, Phys. Rev. Lett. 79, 3660 (1997).

[71] G. Wahnström, Phys. Rev. A 44, 3752 (1991).

[72] W. Kob and H. C. Andersen, Phys. Rev. Lett. 73, 1376 (1994); Transport Theory and 
Statistical Physics 24, 1179 (1995); Phys. Rev. E 52, 4134 (1995).

[73] W. Kob and H. C. Andersen, Phys. Rev. E 51, 4626 (1995).

[74] See, e.g., J. N. Cape and L. V. Woodcock, J. Chem. Phys. 72, 976 (1980).

[75] M. Deserno and C. Holm, preprints cond-mat/9807099 and cond-mat/9807100.

[76] L. Greengard and V. Rokhlin, J. Comp. Phys. 73, 325 (1987); D. Solvason, J. Kolafa, H. G. Petersen, and J. W. Perram, Comp. Phys. Comm. 87, 307 (1995).

[77] J. Horbach, PhD Thesis, University of Mainz, (1998).

[78] J. Horbach, W. Kob, and K. Binder (unpublished).

[79] H. Weber, W. Paul, W. Kob, and K. Binder, Phys. Rev. Lett. 78, 2136 (1997).

[80] C. A. Angell, P. H. Poole, and J. Shao, Nuovo Cimento D 16, 993 (1994).

[81] W. Götze and L. Sjögren, Z. Phys. B 65, 415 (1987).

[82] G. Brébec, R. Seguin, C. Sella, J. Bevenot, J. C. Martin, Acta Metall. 28, 327 (1980); J. C. Mikkelsen, Appl. Phys. Lett. 45, 1187 (1984).

[83] P. Gallo, F. Sciortino, P. Tartaglia and S.-H. Chen, Phys. Rev. Lett. 76, 2730 (1996); S.-H. Chen, P. Gallo, F. Sciortino, and P. Tartaglia, Phys. Rev. E 564231 (1997); F. Sciortino, L. Fabbian, S.-H. Chen, and P. Tartaglia, Phys. Rev. E 565397 (1997).

[84] E. Rössler and A. P. Sokolov, Chemical Geology 128, 143 (1996).

[85] R. J. Speedy, J. Chem. Phys. 107, 3222 (1997).

[86] C. A. Angell, in Water: A Comprehensive Treatise Ed.: F. Franks (Plenum, New York, 1981); E. W. Lang and H. D. Lüdemann, Ber. Bunsenges. Phys. Chem. 85, 603 (1981).

[87] H. Teichler, Phys. Rev. Lett. 76, 62 (1996).

[88] J. H. R. Clarke, p. 272 in Monte Carlo and Molecular Dynamics Simulations in Polymer Science Ed.: K. Binder (Oxford University Press, New York, 1995).

[89] W. Paul and J. Baschnagel, p. 307 in Monte Carlo and Molecular Dynamics Simulations in Polymer Science Ed.: K. Binder (Oxford University Press, New York, 1995).

[90] See, e.g., the articles in J. Res. Nat. Inst. Std. Tech. 102, March-April (1997) and references therein.

[91] M. Wolfgardt, J. Baschnagel, W. Paul, and K. Binder, Phys. Rev. E. 54, 1535 (1996).

[92] L. Sjögren, Phys. Rev. A 22, 2866 (1980) and references therein.

[93] M. Nauroth and W. Kob, Phys. Rev. E 55, 657 (1997).

[94] T. Gleim, W. Kob, and K. Binder, preprint cond-mat/9805200.

[95] H. Löwen, J.-P. Hansen, and J. N. Roux, Phys. Rev. A 44, 1169 (1991).

[96] T. Gleim, PhD Thesis, University of Mainz (1998).

[97] J. Horbach, W. Kob, and K. Binder, preprint cond-mat/9809229.

[98] L. C. E. Struik, Physical Aging in Amorphous Polymers and Other Materials (Elsevier, Amsterdam, 1978); G. B. McKenna, p. 311 in Comprehensive Polymer Science, Vol. 12, Polymer Properties, Eds.: C. Booth and C. Price (Pergamon, Oxford, 1989).

[99] J.-P. Bouchaud, L.F. Cugliandolo, J. Kurchan, and M. Mézard, Physica A 226, 243 (1996); J.-P. Bouchaud, L.F. Cugliandolo, J. Kurchan, and M. Mézard, p. 161 in Ref. [17].

[100] S. Ciuchi and F. de Pasquale, Nuclear Physics B 300, 31 (1988); L. F. Cugliandolo and J. Kurchan, Phys. Rev. Lett. 71, 173 (1993); L. F. Cugliandolo, J. Kurchan, and G. Parisi, J. Phys. I (France) 4, 1641 (1994); J. P. Bouchaud and D. S. Dean, J. Phys. I (France) 5, 265 (1995); C. Monthus and J.-P. Bouchaud, J. Phys. A: Math. Gen. 29, 3847 (1996); H. Yoshino, J. Phys. A: Math. Gen. 30, 1143 (1997); L. F. Cugliandolo 
and J. Kurchan, preprint cond-mat/9807226.

[101] Th. M. Nieuwenhuizen, preprint cond-mat/9807161.

[102] D. S. Fisher and D. A. Huse, Phys. Rev. B 38, 373 (1988); G. J. M. Koper and H. J. Hilhorst, J. Phys. (France) 49, 429 (1988).

[103] L. Lundgren, P. Svedlindh, P. Nordblad, and O. Beckman, Phys. Rev. Lett. 51, 911 (1983); M. Alba, E. Vincent, J. Hammann, and M. Ocio, J. Appl. Phys. 61, 4092 (1987); E. Vincent, J.-P. Bouchaud, D. S. Dean, and J. Hammann, Phys. Rev. B 52, 1050 (1995); C. Djurberg, J. Mattson, and P. Nordblad, Europhys. Lett. 29, 163 (1995).

[104] H. Rieger, J. Phys. A 26, L615, (1993); J. Phys. I (France) 4, 883 (1994); H. Rieger, B. Steckemetz, and M. Schreckenberg, Europhys. Lett. 27, 485 (1994); S. Franz and H. Rieger, J. Stat. Phys. 79, 749 (1995); D. Alvarez, S. Franz, and F. Ritort, Phys. Rev. B 54, 9756 (1996); J. Kisker, L. Santen, M. Schreckenberg, and H. Rieger, Phys. Rev. B 53, 6418 (1996); E. Marinari, G. Parisi, F. Ricci-Tersenghi, and J. J. Ruiz-Lorenzo, J. Phys. A: Math. Gen. 31, 2611 (1998).

[105] A. Barrat, Phys. Rev. E, 55, 5651 (1997); ibid., 57, 3629 (1998); H. Yoshino, preprint cond-mat/9802283 (1998).

[106] P. Chandra, M. V. Feigelman, L. B. Ioffe, and D. M. Kagan, Phys. Rev. B 56, 11553 (1997); L. Frachebourg, P. L. Krapivsky, and S. Redner, Phys. Rev. E 55, 6684 (1997); C. Godrèche and J. M. Luck, J. Phys.: Math. Gen. 30, 6245 (1997); A. Prados, J. J. Brey, and B. Sánchez-Rey, Europhys. Lett. 40, 13 (1997).

[107] F. Alberici, P. Doussineau, and A. Levelut, Europhys. Lett. 39, 329 (1997); E. Salmon, M. Ausloos, and N. Vandewalle, Phys. Rev. E 55, R6348 (1997); R. L. Leheny and S. R. Nagel, Phys. Rev. B 57, 5154 (1998); D. Bonn, H. Tanaka, G. Wegdam, H. Kellay, and J. Meunier, preprint 1998.

[108] W. Kob and J.-L. Barrat, Phys. Rev. Lett. 78, 4581 (1997).

[109] J.-L. Barrat and W. Kob, preprint cond-mat/9806027.

[110] E. Andrejew and J. Baschnagel, Physica A 233, 117 (1996).

[111] W. Kob and J.-L. Barrat, preprint cond-mat/9807364.

[112] J. Baschnagel, Phys. Rev. B 49, 135 (1994).

[113] L. F. Cugliandolo, J. Kurchan, and L. Peliti, Phys. Rev. E 55, 3898 (1997).

[114] A. Q. Tool and C. G. Eichlin, J. Opt. Soc. Amer. 14, 276 (1931).

[115] T. A. Weber and F. H. Stillinger, Phys. Rev. B 31, 1954 (1985); ibid. 32, 5402 (1985); T. Keyes, J. Chem. Phys. 101, 5081 (1994); B. B. Laird and H. R. Schober, Phys. Rev. Lett. 66, 636 (1991); H. R. Schober and B. B. Laird, Phys. Rev. B 44, 6746 (1991); S. Bembenek and B. B. Laird, Phys. Rev. Lett. 74, 936 (1995); J. Chem. Phys. 104, 5199 (1996); J. D. Gezelter, E. Rabani, B. J. Berne, J. Chem. Phys. 107, 4618 (1997); T. Keyes, G. V. Vijayadamodar, and U. Zurcher J. Chem. Phs. 106, 4651 (1997). 


\section{FIGURES}

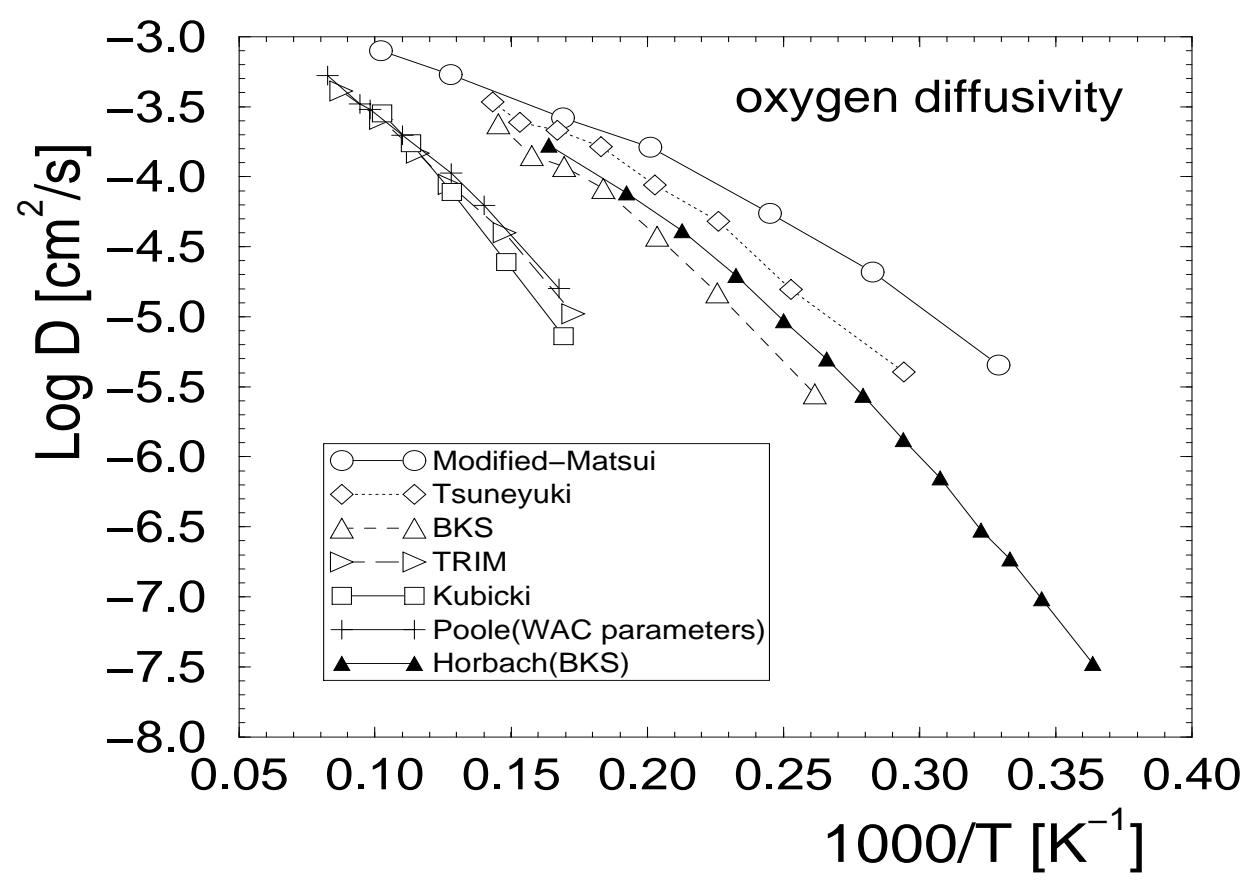

FIG. 1. Temperature dependence of the oxygen diffusion constant for different models of $\mathrm{SiO}_{2}$. See Ref. [61] for details. Adapted from Ref. [61], with permission. 

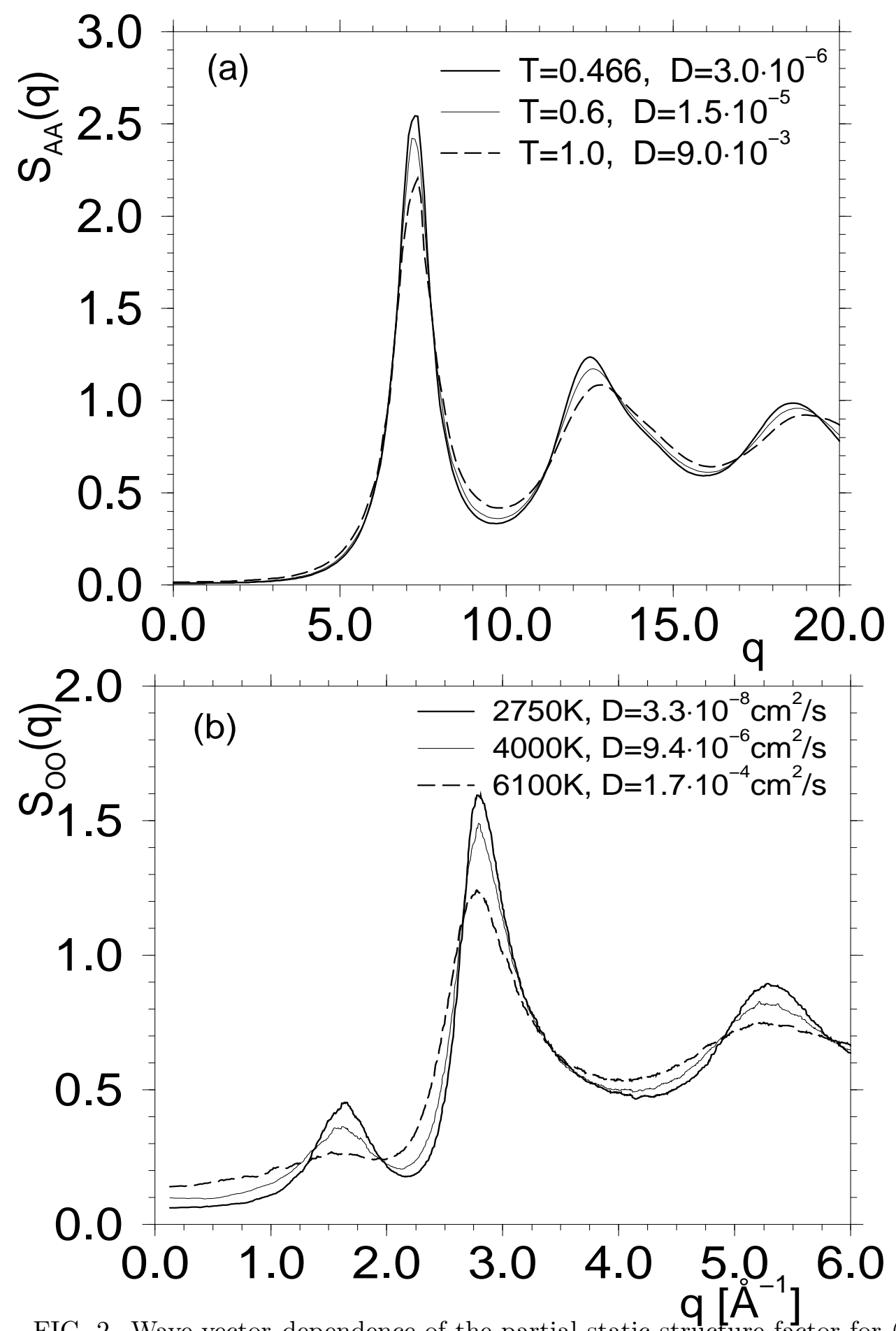

FIG. 2. Wave-vector dependence of the partial static structure factor for (a) a Lennard-Jones system, a fragile glass former, ( $A$ - $A$ correlation) and (b) $\mathrm{SiO}_{2}$, a strong glass former (oxygen-oxygen-correlation). The curves correspond to different temperatures and range from the temperatures at which the system is in a normal liquid state, to temperatures at which it is in a deeply supercooled liquid state. Also given are the values of the diffusion constants for the $A$ particles (a) and the oxygen atoms (b). From Refs. 72,78. 

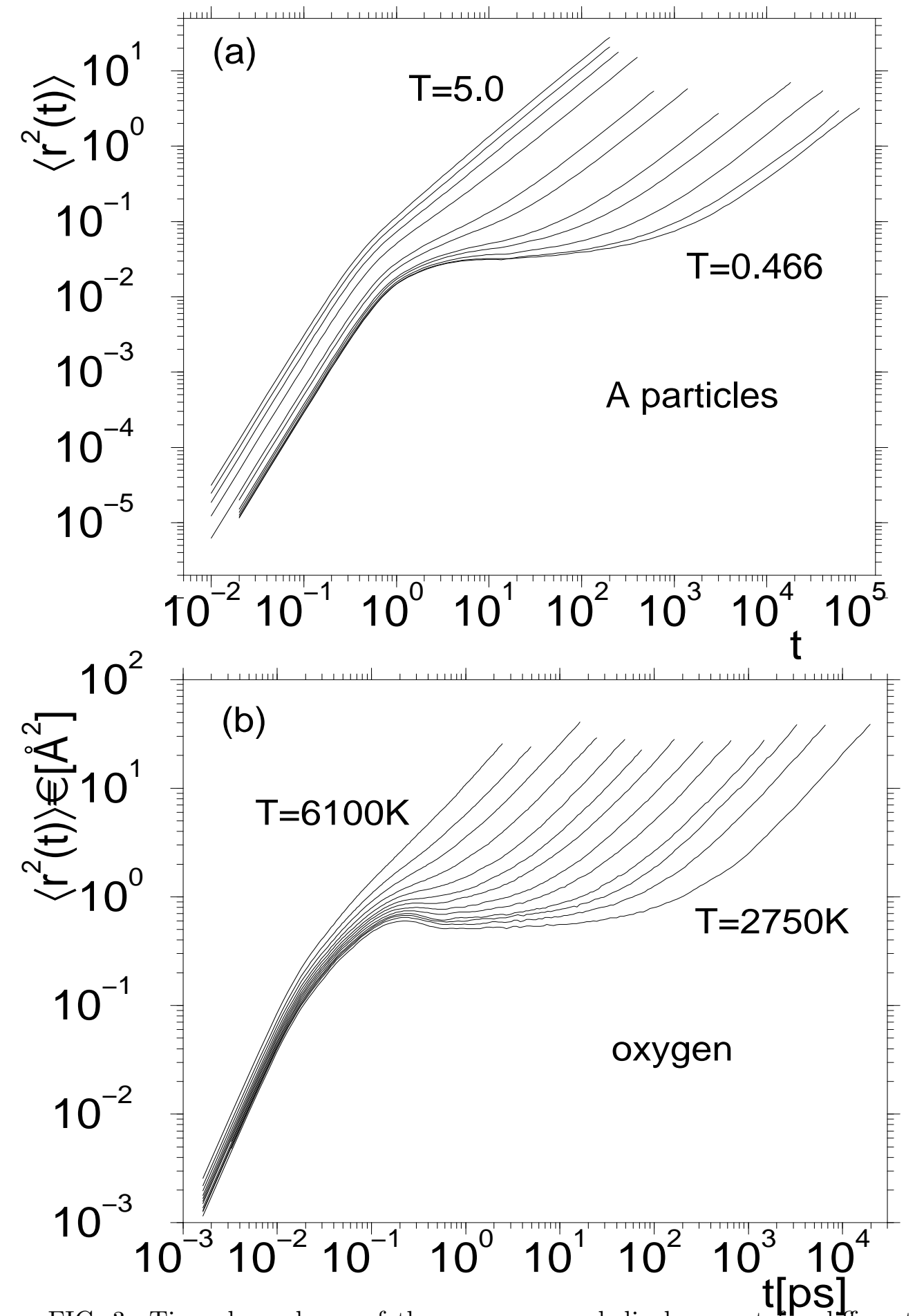

FIG. 3. Time dependence of the mean squared displacement for different temperatures. a) Lennard-Jones system. $T=5.0,4.0,3.0,2.0,1.0,0.8,0.6,0.55,0.5,0.475,0.466$. b) Silica. $T=6100 \mathrm{~K}, 5200 \mathrm{~K}, 4700 \mathrm{~K}, 4300 \mathrm{~K}, 4000 \mathrm{~K}, 3760 \mathrm{~K}, 3580 \mathrm{~K}, 3400 \mathrm{~K}, 3250 \mathrm{~K}, 3100 \mathrm{~K}, 3000 \mathrm{~K}$ and $2900 \mathrm{~K}$ and $2750 \mathrm{~K}$. From Refs. 64, 73]. 


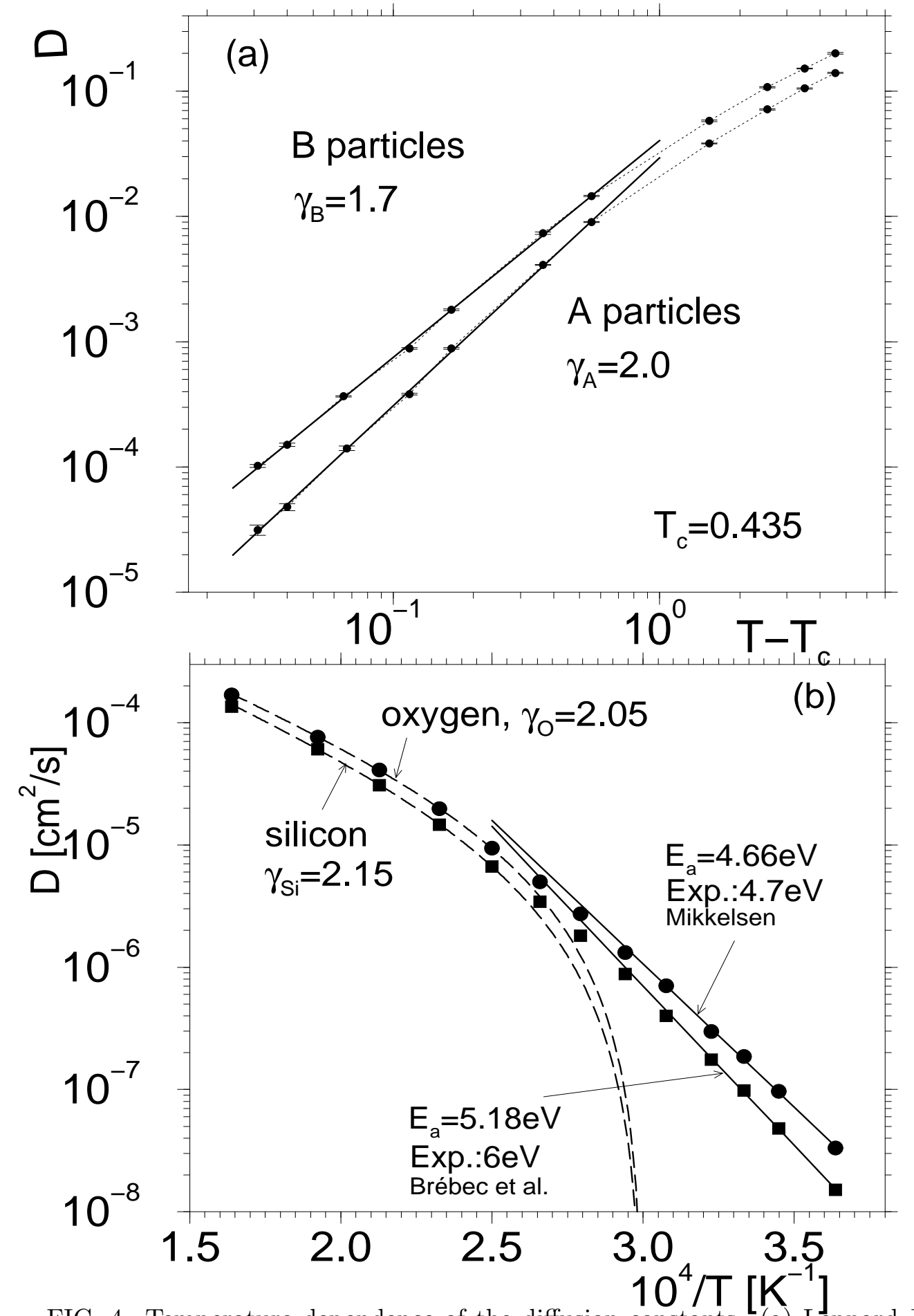

FIG. 4. Temperature dependence of the diffusion constants. (a) Lennard-Jones system. The solid lines are fits with a power-law of the form $D \propto\left(T-T_{c}\right)^{\gamma}$. (b) Silica. The solid lines are fits with an Arrhenius law with the stated activation energies. The experimental values are from Refs. 82. The dashed lines are power-law with $T_{c}=3330 \mathrm{~K}$. From Refs. 64, 72, 

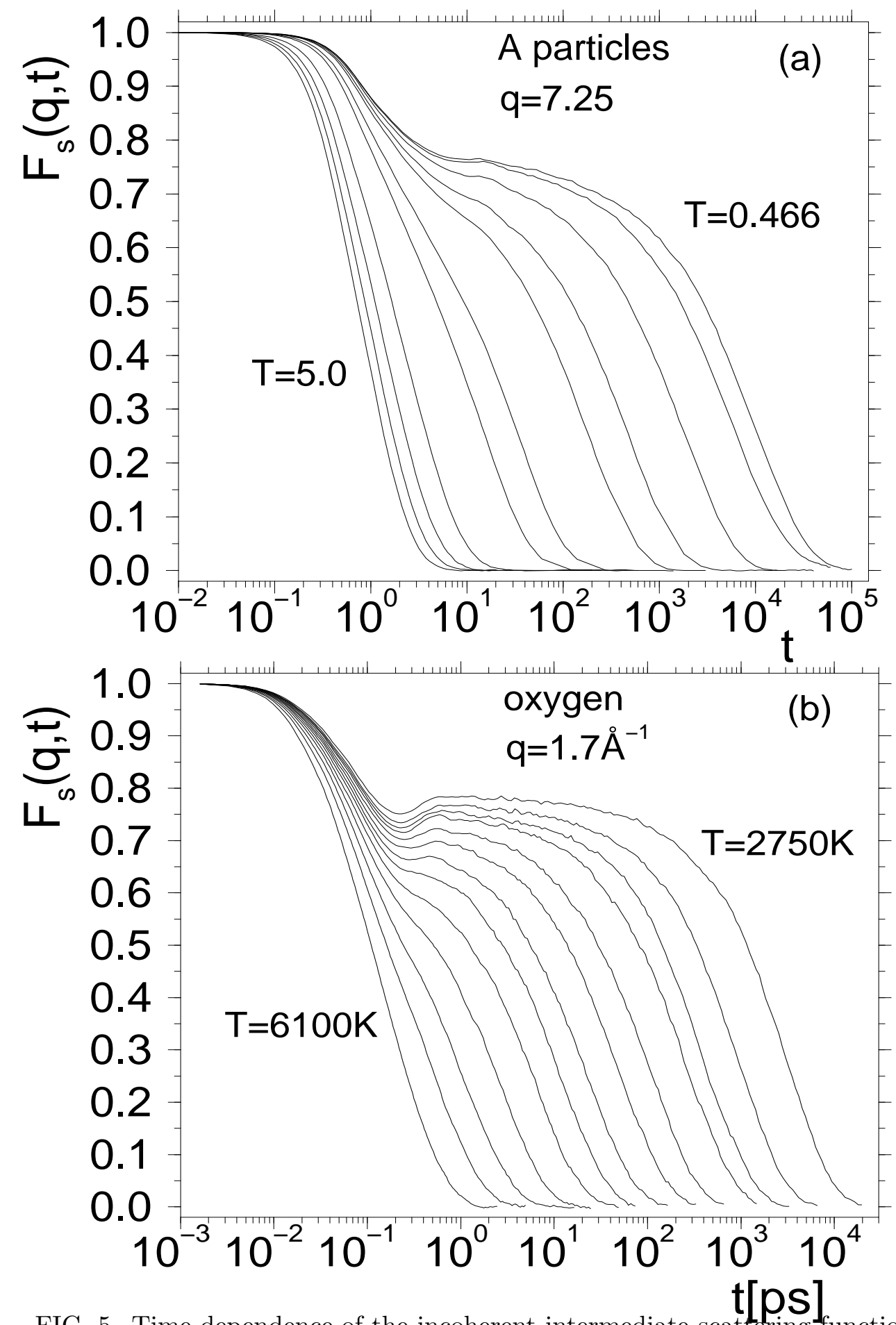

FIG. 5. Time dependence of the incoherent intermediate scattering function for different temperatures (see Fig. 3). a) Lennard-Jones system. b) Silica. From Refs. 64,72, 

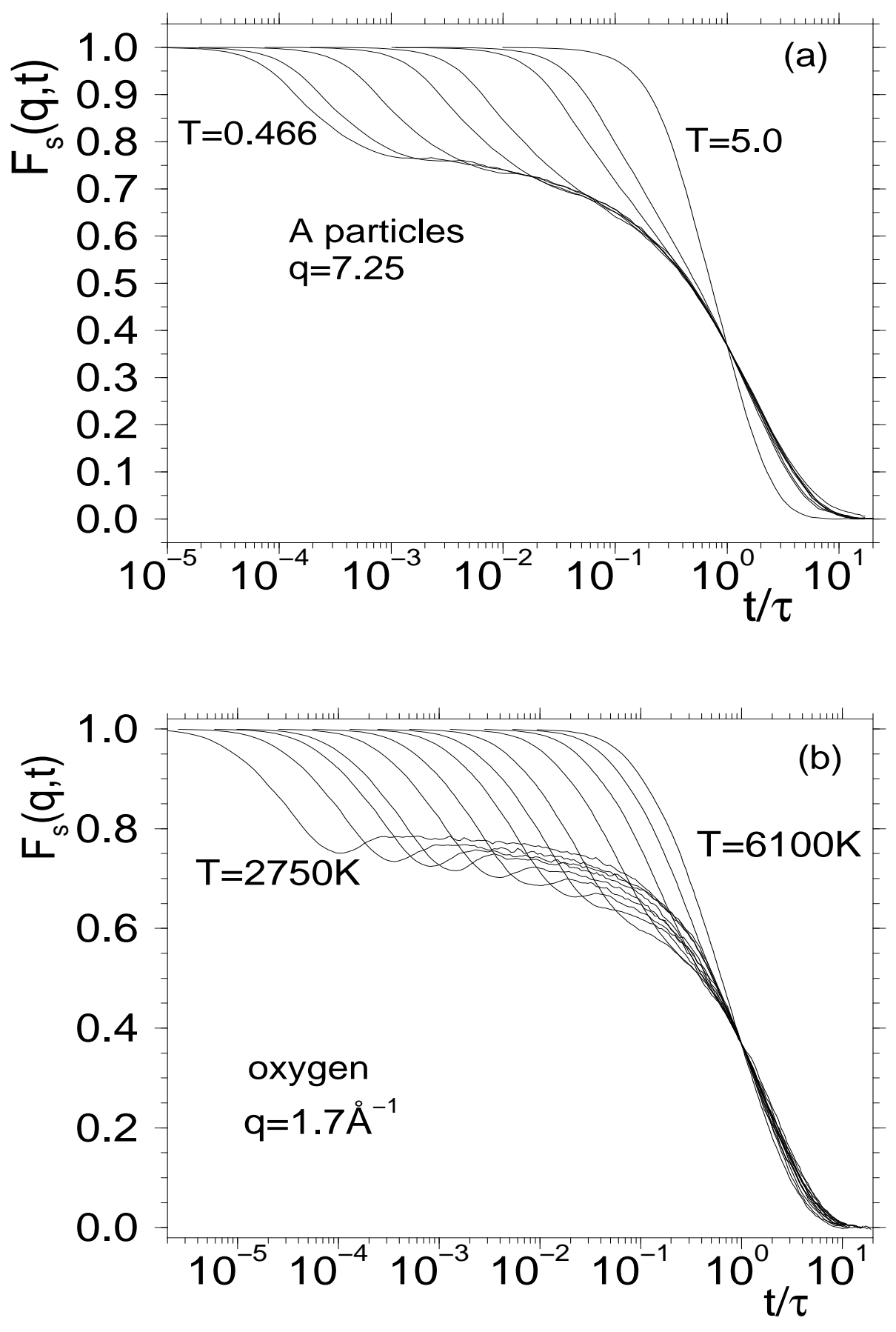

FIG. 6. Incoherent intermediate scattering function versus rescaled time for different temperatures (see Fig. 3). a) Lennard-Jones system. The curves for $T=4.03 .0$, and 2.0 are not shown. b) Silica. From Refs. 64,72. 


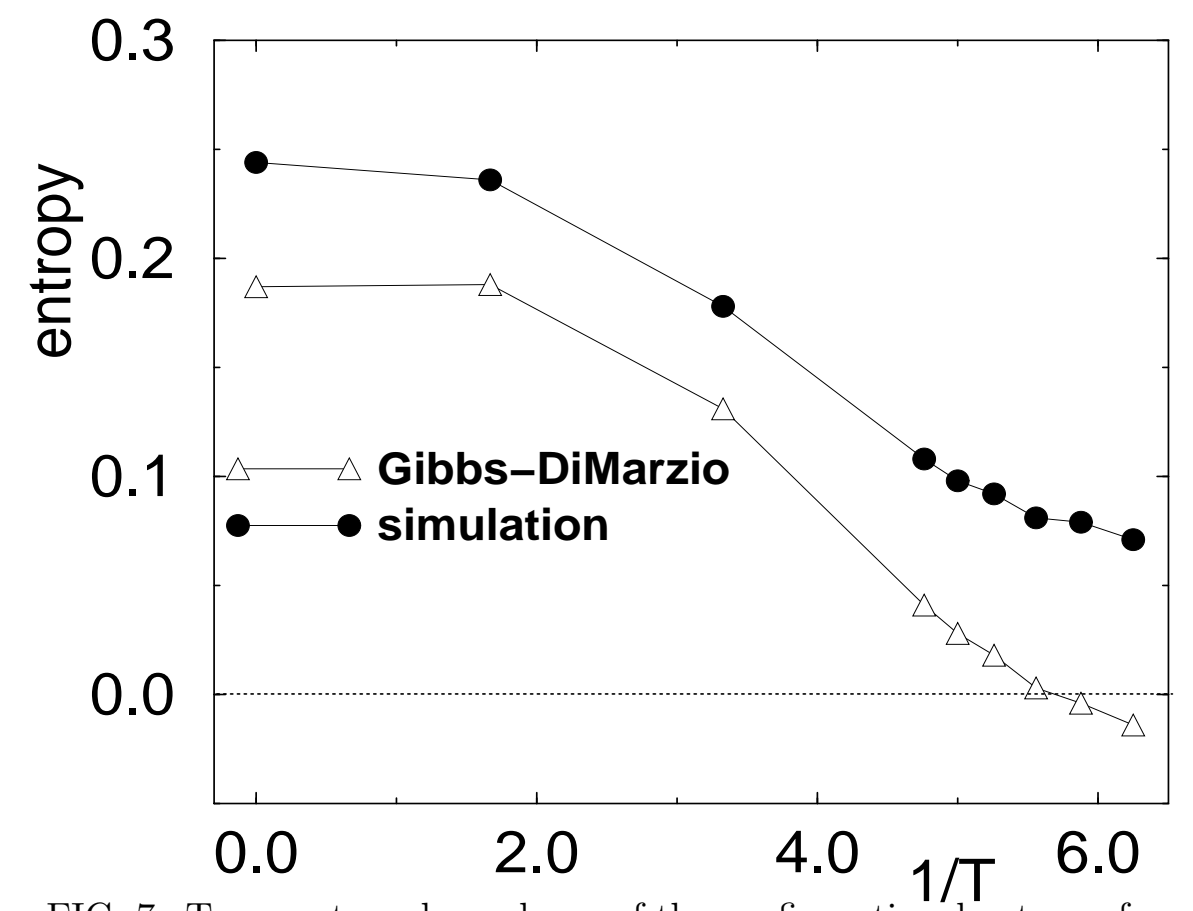

FIG. 7. Temperature dependence of the configurational entropy for a polymer model as determined from a Monte Carlo simulation (filled symbols) and from the prediction of the theory by Gibbs and DiMarzio (open symbols). Adapted from Ref. 91.

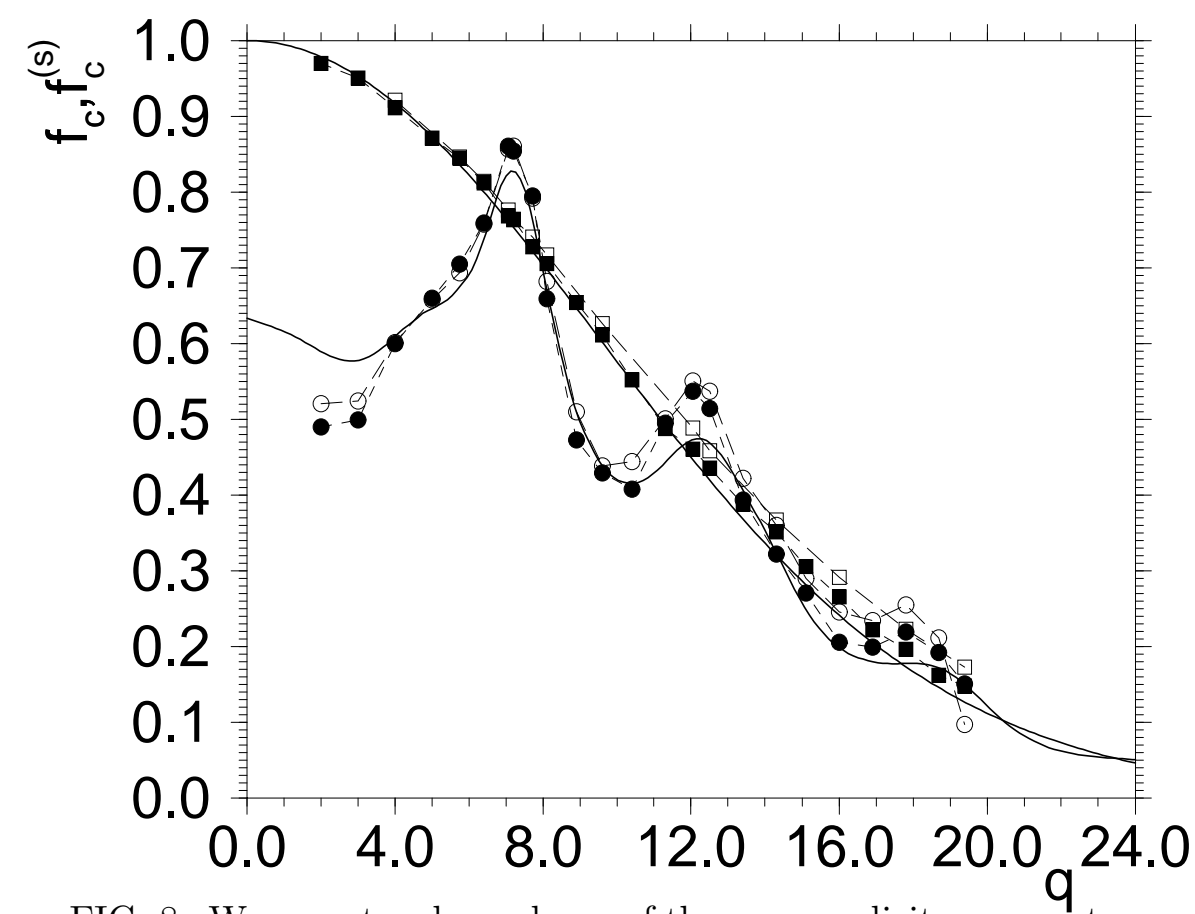

FIG. 8. Wave-vector dependence of the nonergodicity parameter as predicted from MCT (solid lines), as measures in a Lennard-Jones system with Newtonian dynamics (open symbols) and a stochastic dynamics (filled symbols). The Gaussian shaped curves are for the incoherent intermediate scattering function and the oscillatory curves are for the coherent intermediate scattering function. From Ref. [94]. 


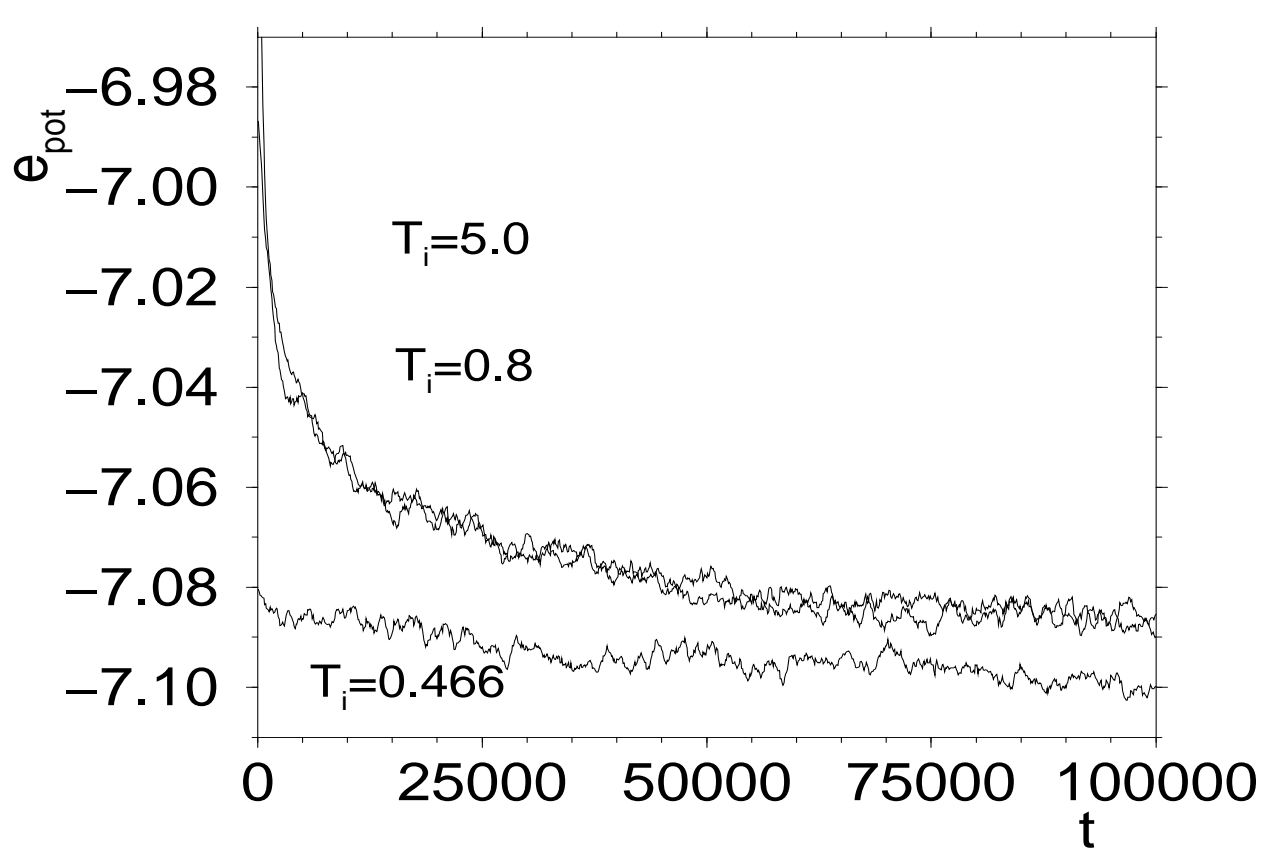

FIG. 9. Time dependence of the potential energy of a Lennard-Jones system which at time zero has been quenched from an initial temperature $T_{i}$ to a final temperature $T_{f}=0.4$. Adapted from Ref. [108].

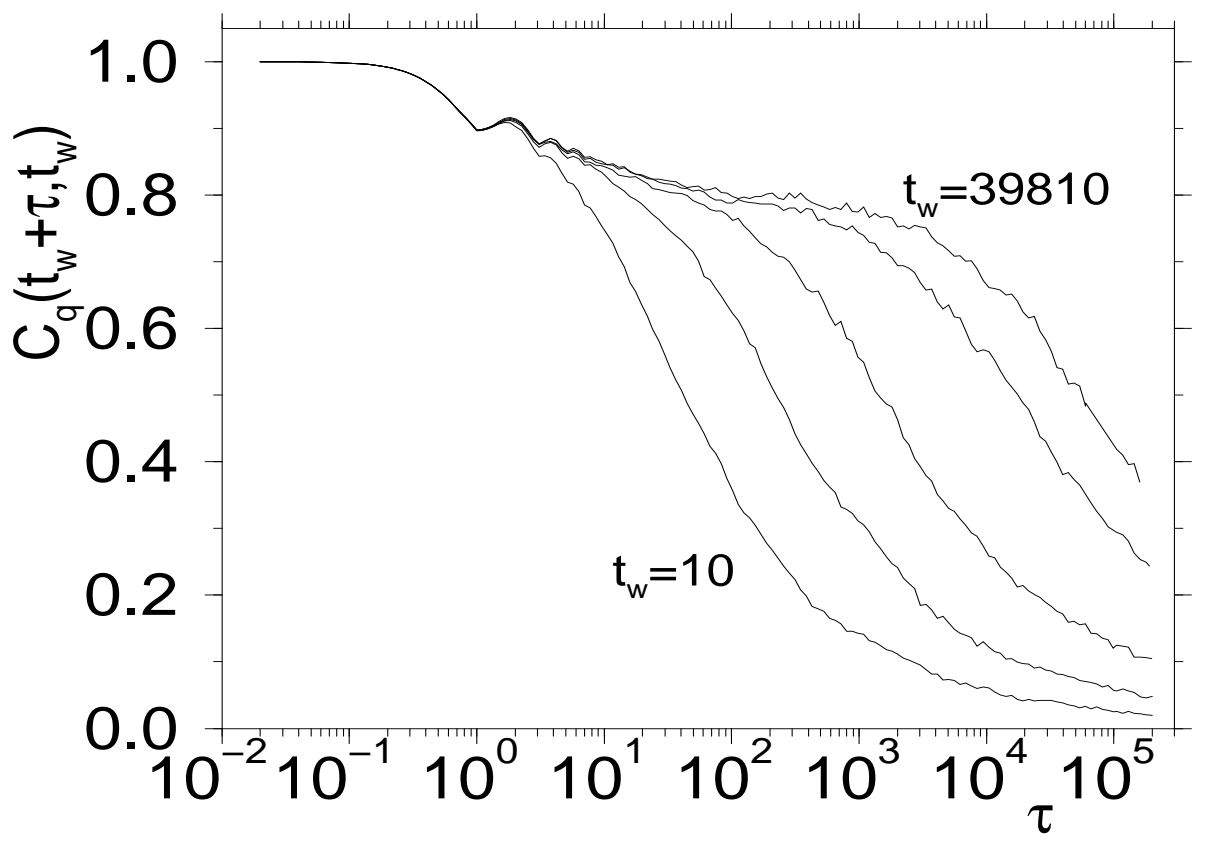

FIG. 10. Time dependence of the out of equilibrium correlation function $C_{q}\left(t_{w}+\tau, t_{w}\right)$ for different waiting times $t_{w}$. From left to right: $t_{w}=10,100,1000,10000$ and 39810. The wave-vector $q$ is 7.25 , the location of the maximum in the static structure factor. Adapted from Ref. [108]. 


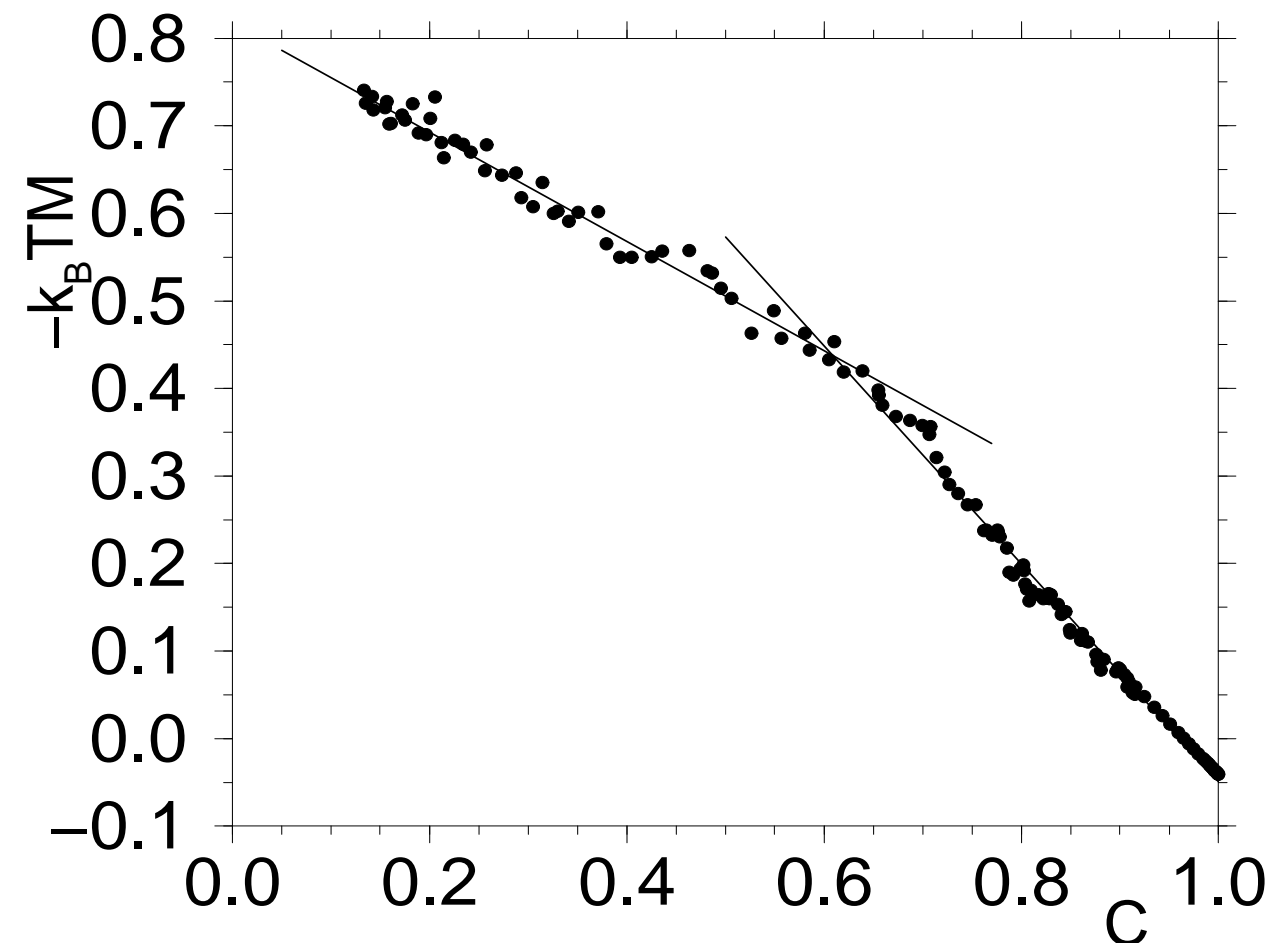

FIG. 11. Parametric plot of the integrated response $M$ versus the tho-time correlation function $C_{q}$. The two straight lines have slopes around -0.6 and -1 , respectively. Adapted from Ref. [111]. 